\title{
The HERMES Polarized Hydrogen and Deuterium Gas Target in the HERA Electron Storage Ring
}

The HERMES Collaboration

\begin{abstract}
A. Airapetian $^{\text {r }}$, N. Akopov ad, Z. Akopov ad, M. Amarian ${ }^{\text {h,ad }}$, A. Andrus ${ }^{\text {p }}$, E.C. Aschenauer ${ }^{\text {h }}$, W. Augustyniak ${ }^{\text {ac }}$, R. Avakian ${ }^{\text {ad }}$, A. Avetissian ${ }^{\text {ad }}$, E. Avetissian ${ }^{\ell}$, P. Bailey ${ }^{\mathrm{p}}$, D. Balin ${ }^{u}$, C. Baumgarten ${ }^{\text {ae,aj, }}$ M. Beckmann ${ }^{\mathrm{g}}$, S. Belostotski ${ }^{\mathrm{u}}$, N. Bianchi ${ }^{\ell}$, H.P. Blok ${ }^{\mathrm{t}, \mathrm{ab}}$, H. Böttcher ${ }^{\mathrm{h}}$, A. Borissov ${ }^{\mathrm{o}}$, A. Borysenko $^{\ell}$, M. Bouwhuis $^{p}$, B. Braun ${ }^{j}$, A. Brüll ${ }^{q}$, V. Bryzgalov ${ }^{v}$, G.P. Capitani ${ }^{\ell}$, M. Capiluppi ${ }^{\mathrm{k}}$, T. Chen ${ }^{\mathrm{d}}$, G. Ciullo $^{k}$, M. Contalbrigo ${ }^{k}$, G. Court ${ }^{\text {af }}$, P.F. Dalpiaz ${ }^{k}$, R. De Leo ${ }^{\mathrm{c}}$, M. Demey ${ }^{\mathrm{t}}$, L. De Nardo ${ }^{\mathrm{a}}$, E. De Sanctis ${ }^{\ell}$, E. Devitsin ${ }^{\text {s }}$, P. Di Nezza ${ }^{\ell}$, M. Düren ${ }^{n}$, M. Ehrenfried ${ }^{j}$, A. Elalaoui-Moulay ${ }^{\text {b }}$, G. Elbakian ${ }^{\text {ad }}$, F. Ellinghaus ${ }^{\text {h }}$, U. Elschenbroich $^{\mathrm{m}}$, R. Fabbri ${ }^{\mathrm{t}}$, A. Fantoni ${ }^{\ell}$, A. Fechtchenko $^{\mathrm{i}}$, L. Felawka ${ }^{\text {, S. Srullani }}{ }^{\mathrm{x}}$, G. Gapienko ${ }^{\mathrm{v}}$, V. Gapienko ${ }^{\mathrm{v}}$, F. Garibaldi ${ }^{x}$, K. Garrow ${ }^{z}$, G. Gavrilov ${ }^{g, z}$, V. Gharibyan ${ }^{\text {ad }}$, G. Graw ${ }^{\text {ae }}$, O. Grebeniouk ${ }^{u}$, I.M. Gregor ${ }^{\text {h }}$, C. Hadjidakis ${ }^{\ell}$, W. Haeberli ${ }^{\text {ag }}$, K. Hafidi ${ }^{\mathrm{b}}$, M. Hartig ${ }^{\mathrm{n}}$, D. Hasch ${ }^{\ell}$, D. Heesbeen ${ }^{\mathrm{t}}$, M. Henoch ${ }^{\mathrm{j}}$, R. Hertenberger ${ }^{\mathrm{ae}}$, W.H.A. Hesselink ${ }^{\mathrm{t}, \mathrm{ab}}$, A. Hillenbrand ${ }^{\mathrm{j}}$, M. Hoek ${ }^{\mathrm{n}}$, Y. Holler ${ }^{\mathrm{g}}$, B. Hommez ${ }^{\mathrm{m}}$, I. Hristova ${ }^{\mathrm{h}}$, G. Iarygin $^{\mathrm{i}}$, A. Ivanilov ${ }^{\mathrm{v}}$, A. Izotov ${ }^{u}$, H.E. Jackson ${ }^{\text {b }}$, A. Jgoun ${ }^{\text {u }}$, R. Kaiser ${ }^{\circ}$, E. Kinney ${ }^{f}$, A. Kisselev ${ }^{\mathrm{f}}$, T. Kobayashi ${ }^{\text {aa }}$, N. Koch ${ }^{\mathrm{j}, \text { ah }}$, H. Kolster ${ }^{\text {ab,ae,ai, }}$ M. Kopytin ${ }^{\text {h, V. Korotkov }}{ }^{v}$, V. Kozlov ${ }^{\mathrm{s}}$, B. Krauss ${ }^{\mathrm{j}}$, V.G. Krivokhijine ${ }^{\mathrm{i}}$, L. Lagamba ${ }^{\mathrm{c}}$, L. Lapikás ${ }^{\mathrm{t}}$, A. Laziev ${ }^{\mathrm{t}, \mathrm{ab}}$, P. Lenisa ${ }^{\mathrm{k}, 1}$, P. Liebing ${ }^{\mathrm{h}}$, L.A. Linden-Levy ${ }^{\mathrm{p}}, \mathrm{W}$. Lorenzon ${ }^{\mathrm{r}}$, H. $\mathrm{Lu}^{\mathrm{e}}$, J. $\mathrm{Lu}^{\mathrm{z}}, \mathrm{S} . \mathrm{Lu}^{\mathrm{n}}$, B.-Q. Ma ${ }^{\mathrm{d}}$, B. Maiheu ${ }^{\mathrm{m}}$, N.C.R. Makins ${ }^{\mathrm{p}}$, Y. Mao ${ }^{\mathrm{d}}$, B. Marianski ${ }^{\text {ac }}$, H. Marukyan $^{\text {ad }}$, V. Mexner ${ }^{\mathrm{t}}$, N. Meyners ${ }^{\mathrm{g}}$, R. Mussa ${ }^{\mathrm{k}}$, O. Mikloukho ${ }^{\mathrm{u}}$,
\end{abstract}


C.A. Miller ${ }^{\text {a,z }}$, Y. Miyachi ${ }^{\text {aa }}$, V. Muccifora ${ }^{\ell}$, A. Nagaitsev $^{\mathrm{i}}$, E. Nappi ${ }^{\mathrm{c}}$, Y. Naryshkin ${ }^{\mathrm{u}}$, A. Nass ${ }^{\mathrm{j}}$, M. Negodaev $^{\mathrm{h}}$, W.-D. Nowak ${ }^{\mathrm{h}}$, K. Oganessyan ${ }^{\mathrm{g}, \ell}, \mathrm{H}$. Ohsuga ${ }^{\text {aa }}$, A. Osborne ${ }^{\mathrm{a}}$, N. Pickert ${ }^{j}$, D.H. Potterveld ${ }^{b}$, M. Raithel ${ }^{j}$, D. Reggiani ${ }^{k}$, P.E. Reimer ${ }^{\mathrm{b}}$, A. Reischl ${ }^{\mathrm{t}}$, A.R. Reolon ${ }^{\ell}, \mathrm{C}$ Riedl $^{\mathrm{j}}, \mathrm{K}^{\mathrm{K}}$ Rith $^{\mathrm{j}}$, G. Rosner ${ }^{\circ}$, A. Rostomyan ${ }^{\text {ad }}$, L. Rubacek ${ }^{\mathrm{n}}$, J. Rubin ${ }^{\mathrm{p}}$, D. Ryckbosch ${ }^{\mathrm{m}}$, Y. Salomatin ${ }^{\mathrm{v}}$, I. Sanjiev ${ }^{\mathrm{b}, \mathrm{u}}$, I. Savin ${ }^{\mathrm{i}}$, C. Schill ${ }^{\ell}$, G. Schnell ${ }^{\text {h}}$, K.P. Schüler ${ }^{g}$, J. Seele ${ }^{p}$, R. Seidl ${ }^{j}$, B. Seitz ${ }^{n}$, R. Shanidze ${ }^{j}$, C. Shearer ${ }^{\circ}$, T.-A. Shibata ${ }^{\text {aa, }}$, V. Shutov ${ }^{i}$, K. Sinram ${ }^{\mathrm{g}}$, W. Sommer ${ }^{n}$, M. Stancari ${ }^{\mathrm{k}}$, M. Statera ${ }^{k}$, E. Steffens ${ }^{j}$, J.J.M. Steijger ${ }^{\mathrm{t}}$, H. Stenzel ${ }^{\mathrm{n}}$, J. Stewart ${ }^{\text {h }}$, F. Stinzing ${ }^{j}$, P. Tait ${ }^{j}$, H. Tanaka ${ }^{\text {aa }}$, S. Taroian ${ }^{\text {ad }}$, B. Tchuiko ${ }^{\mathrm{v}}$, A. Terkulov ${ }^{\mathrm{s}}$, A. Trzcinski ${ }^{\text {ac }}$, M. Tytgat ${ }^{\mathrm{m}}$, A. Vandenbroucke ${ }^{\mathrm{m}}, \mathrm{P}$. van der Nat ${ }^{\mathrm{t}}$, G. van der Steenhoven ${ }^{\mathrm{t}}$, Y. van Haarlem ${ }^{\mathrm{m}}$, M.C. Vetterli ${ }^{\mathrm{y}, \mathrm{z}}$, V. Vikhrov ${ }^{\mathrm{u}}$, M.G. Vincter ${ }^{\mathrm{a}}$, C. Vogel $^{\mathrm{j}}$, J. Volmer ${ }^{\mathrm{h}}$, S. Wang ${ }^{\mathrm{e}}$, J. Wendland ${ }^{\mathrm{y}, \mathrm{z}}$, J. Wilbert ${ }^{\mathrm{j}}$, T. Wise ${ }^{\mathrm{ag}}$, G. Ybeles Smit ${ }^{\mathrm{ab}}$, Y. Ye ${ }^{\mathrm{e}}, \mathrm{Z} \mathrm{Ye}^{\mathrm{e}}, \mathrm{S}$ Yen $^{\mathrm{z}}$, B. Zihlmann ${ }^{\mathrm{m}}$, P. Zupranski ${ }^{\text {ac }}$

${ }^{a}$ Department of Physics, University of Alberta, Edmonton, Alberta T6G 2J1, Canada

b Physics Division, Argonne National Laboratory, Argonne, Illinois 60439-4843, $U S A$

${ }^{\mathrm{c}}$ Istituto Nazionale di Fisica Nucleare, Sezione di Bari, 70124 Bari, Italy

${ }^{\mathrm{d}}$ School of Physics, Peking University, Beijing 100871, China

${ }^{\mathrm{e}}$ Department of Modern Physics, University of Science and Technology of China, Hefei, Anhui 230026, China

${ }^{\mathrm{f}}$ Nuclear Physics Laboratory, University of Colorado, Boulder, Colorado 80309-0446, USA

g DESY, 22603 Hamburg, Germany

${ }^{\mathrm{h}}$ DESY, 15738 Zeuthen, Germany

i Joint Institute for Nuclear Research, 141980 Dubna, Russia

j Physikalisches Institut, Universität Erlangen-Nürnberg, 91058 Erlangen, Germany

${ }^{\mathrm{k}}$ Istituto Nazionale di Fisica Nucleare, Sezione di Ferrara and Dipartimento di Fisica, Università di Ferrara, 44100 Ferrara, Italy

${ }^{\ell}$ Istituto Nazionale di Fisica Nucleare, Laboratori Nazionali di Frascati, 00044 Frascati, Italy 
${ }^{\mathrm{m}}$ Department of Subatomic and Radiation Physics, University of Gent, 9000 Gent, Belgium

${ }^{\mathrm{n}}$ Physikalisches Institut, Universität Gießen, 35392 Gießen, Germany

${ }^{\circ}$ Department of Physics and Astronomy, University of Glasgow, Glasgow G12 $8 Q Q$, United Kingdom

${ }^{\mathrm{p}}$ Department of Physics, University of Illinois, Urbana, Illinois 61801-3080, USA

${ }^{\mathrm{q}}$ Laboratory for Nuclear Science, Massachusetts Institute of Technology, Cambridge, Massachusetts 02139, USA

${ }^{\mathrm{r}}$ Randall Laboratory of Physics, University of Michigan, Ann Arbor, Michigan 48109-1120, USA

${ }^{\mathrm{s}}$ Lebedev Physical Institute, 117924 Moscow, Russia

${ }^{\mathrm{t}}$ Nationaal Instituut voor Kernfysica en Hoge-Energiefysica (NIKHEF), 1009 DB Amsterdam, The Netherlands

${ }^{u}$ Petersburg Nuclear Physics Institute, St. Petersburg, Gatchina, 188350 Russia

' Institute for High Energy Physics, Protvino, Moscow region, 142281 Russia

${ }^{\mathrm{w}}$ Institut für Theoretische Physik, Universität Regensburg, 93040 Regensburg, Germany

${ }^{\mathrm{x}}$ Istituto Nazionale di Fisica Nucleare, Sezione Roma 1, Gruppo Sanità and Physics Laboratory, Istituto Superiore di Sanità, 00161 Roma, Italy

${ }^{y}$ Department of Physics, Simon Fraser University, Burnaby, British Columbia V5A 1S6, Canada

z TRIUMF, Vancouver, British Columbia V6T 2A3, Canada

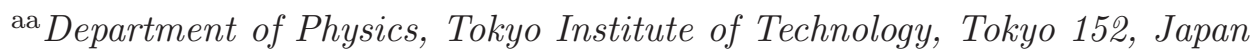

${ }^{\mathrm{ab}}$ Department of Physics and Astronomy, Vrije Universiteit, $1081 \mathrm{HV}$ Amsterdam, The Netherlands

${ }^{a c}$ Andrzej Soltan Institute for Nuclear Studies, 00-689 Warsaw, Poland

ad Yerevan Physics Institute, 375036 Yerevan, Armenia

ae Sektion Physik, Universität München, 85748 Garching, Germany

af Physics Department, University of Liverpool, Liverpool L69 7ZE, United Kingdom

ag Department of Physics, University of Wisconsin-Madison, Madison, Wisconsin 53706 USA

${ }^{\text {ah }}$ Current address: Thales Electron Devices GmbH, Söflinger Str. 100, 8907r Ulm, Germany

ai Current address: Laboratory for Nuclear Science, Massachusetts Institute of Technology, Cambridge, Massachusetts 02139, USA

${ }^{\text {aj }}$ Current address: ACCEL Instruments GmbH, Friedrich-Ebert-Str. 1, D-51429 Bergisch Gladbach, Germany 


\begin{abstract}
The HERMES hydrogen and deuterium nuclear-polarized gas targets have been in use since 1996 with the polarized electron beam of HERA at DESY to study the spin structure of the nucleon. Polarized atoms from a Stern-Gerlach Atomic Beam Source are injected into a storage cell internal to the HERA electron ring. Atoms diffusing from the center of the storage cell into a side tube are analyzed to determine the atomic fraction and the atomic polarizations. The atoms have a nuclear polarization, the axis of which is defined by an external magnetic holding field. The holding field was longitudinal during 1996-2000, and was changed to transverse in 2001. The design of the target is described, the method for analyzing the target polarization is outlined, and the performance of the target in the various running periods is presented.
\end{abstract}

\title{
1 Introduction
}

The HERMES experiment at HERA (DESY, Hamburg) was designed to study the quark-gluon spin structure of the nucleon by deeply inelastic scattering (DIS) of longitudinally polarized electrons from an polarized gas target of hydrogen and deuterium atoms internal to a storage ring[1,2]. The $27.5 \mathrm{GeV}$ electron beam stored in the HERA storage ring with currents up to $50 \mathrm{~mA}$ is polarized by the Sokolov-Ternov effect [3], with a time constant of about 30 minutes. A longitudinal spin direction is obtained where the experiment is located within the HERA east straight section by means of a pair of spin rotators [4]. In a machine with one pair of spin rotators before the 2001 upgrade, a typical polarization of $60 \%$ was achieved.

The HERMES experiment [5] was proposed in 1990, and is designed to run in parallel with the ZEUS and H1 collider experiments without significantly reducing the electron beam lifetime, which is of the order of 10 hours. This limits the target areal density to about $10^{15} \mathrm{H}$-atoms $/ \mathrm{cm}^{2}$ and excludes the use of any solid target material. A target employing a cold storage cell fed by a a polarized atomic beam source was proposed and implemented. Polarized gas targets for storage rings were reviewed recently by Steffens and Haeberli [6].

Since 1996, the present target apparatus for polarized hydrogen and deuterium has been in operation. During the years 1996-97 longitudinally polarized hydrogen $\left(H_{\|}\right)$was used, while longitudinally polarized deuterium was employed in 1998-2000 $\left(D_{\|}\right)$, and from 2002 onwards, transversely polarized hydrogen $\left(H_{\perp}\right)$ has been in use.

$\overline{1}$ Corresponding author: Tel:+49-40-89981963; e-mail: Paolo.Lenisa@desy.de 
Apart from a high areal density and reliable operation over many years, the most challenging requirement for the internal target was to determine the effective target polarization seen by the beam. The target polarization cannot be measured by making use of scattering processes involving the high-energy electron beam due to the small cross sections involved. Nuclear vector polarization $P_{z}$ and, for deuterium also the tensor polarization $P_{z z}$ were to be measured, as well as the electron polarization $P_{e}$ for diagnostic purposes. The required accuracy was $\delta P=0.03$ absolute uncertainty on the average polarization over long measuring periods, and $1 \%$ statistical accuracy within a couple of minutes in order to monitor possible changes in time. This goal was achieved by employing a sampling technique in conjunction with detailed simulations and frequent calibration measurements.

In the present article, an overview of the HERMES polarized hydrogen and deuterium gas target is given with reference to the various papers describing the subsystems of the target. The atomic beam source (ABS) [8], the storage cell [9], the Target Gas Analyzer (TGA) [10] and the Breit-Rabi polarimeter (BRP) [11] are summarized in Section 2. Section 3 describes the analysis applied to the raw TGA and BRP signals and the several calibrations required to derive measured values for the degree of dissociation and the atomic polarizations of the sample gas ${ }^{2}$. Section 4 reviews the various physical processes inside the storage cell that directly influence the TGA and BRP analyses $[12,13,14,15]$. The results of the target analysis are reported in Sections 6 and 7. Section 8 mentions a selection of measurements carried out by HERMES that crucially depended on the polarized target. The paper is concluded in Section 9.

\section{Survey of experimental apparatus}

The HERMES polarized hydrogen and deuterium target, schematically shown in Fig. 1, consists of an atomic beam source (ABS) that injects a spin polarized beam of $\mathrm{H}$ or $\mathrm{D}$ atoms into a thin-walled storage cell. A sample of gas diffuses from the center of the cell into a Breit Rabi Polarimeter (BRP) that measures the atomic polarizations, or into a Target Gas Analyzer (TGA) that measures the relative atomic and molecular content of the gas. A magnet surrounding the storage cell provides a holding field defining the polarization axis and preventing spin relaxation by effectively decoupling the magnetic moments of electrons and nucleons.

$\overline{2}$ In this article, the term "atomic polarizations" refers to the polarizations of the electrons and nuclei in the atoms 


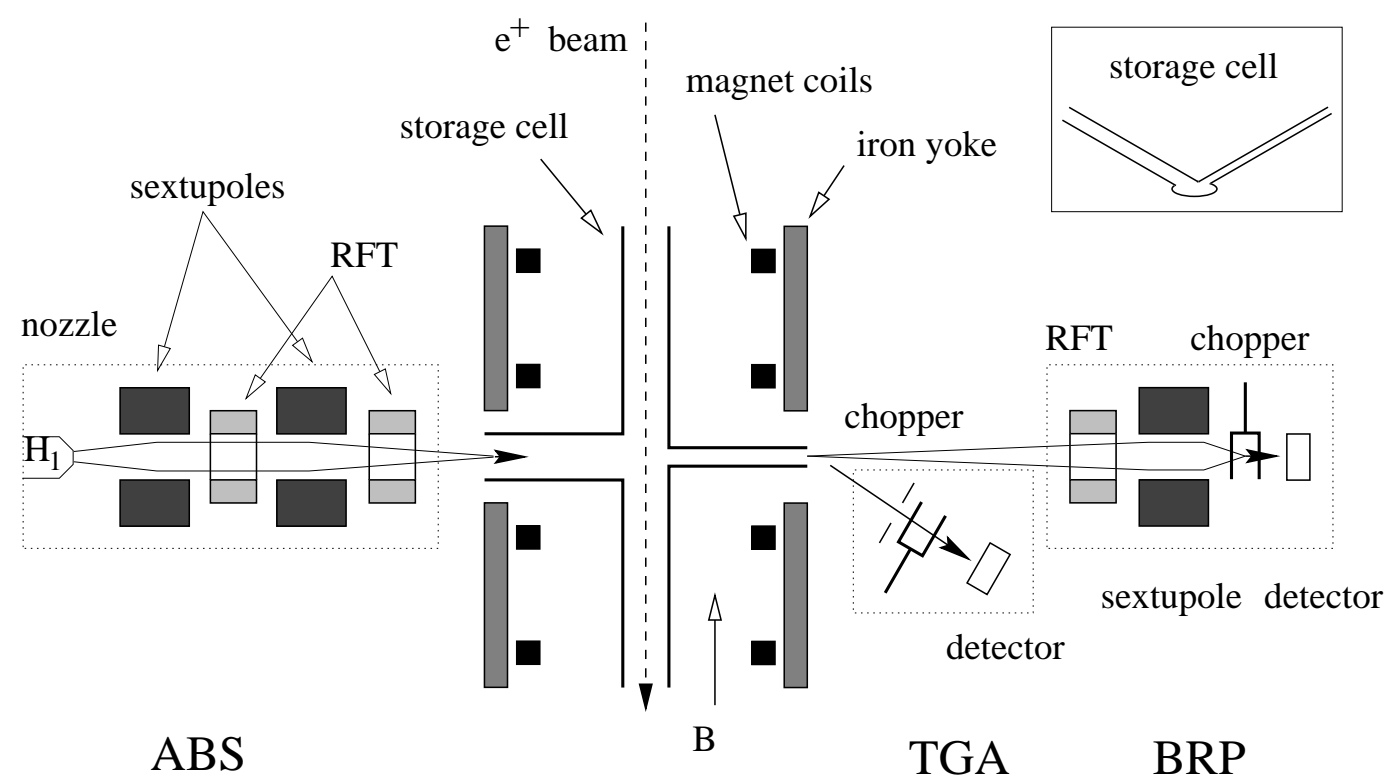

Fig. 1. Schematic view of the HERMES longitudinally polarized target. From left to right: Atomic Beam Source (ABS), target chamber with cell and magnet, and diagnostic system composed by Target Gas Analyzer (TGA) and Breit-Rabi Polarimeter (BRP). The locations of the radio-frequency transition (RFT) units are indicated.

\subsection{Target chamber}

The target chamber shown in Fig. 2 is evacuated by two turbo-molecular pumps with a combined speed of $4400 \mathrm{ls}^{-1}$. During operation the chamber pressure is typically in the $10^{-7}$ mbar range due to the high gas load from the atomic beam, which enters the chamber from $30^{\circ}$ above the horizontal plane. The storage cell and its supporting cooling rails are cantilevered from the upstream ${ }^{3}$ end flange.

A $0.3 \mathrm{~mm}$ thick stainless steel exit window on the downstream end of the target chamber allows the scattered electrons and hadronic fragments to leave the target chamber and to be detected by the HERMES spectrometer [5]. This thin window is integral with the following thin-walled beam tube with no intervening flange, in order to minimize the high- $Z$ material in this region and reduce shower production by $\pi^{0}$ mesons produced at small angles. About $1 \mathrm{~m}$ upstream of the target cell, an adjustable collimator (labeled $\mathrm{C} 1$ ) is located to stop direct synchrotron radiation. It normally has the smallest scaled aperture in the HERA ring, and is opened during electron injection [5]. A fixed collimator (labeled C2) just upstream of the storage cell protects it from synchrotron light scattered by $\mathrm{C} 1$ and from exposure to electron showers from $\mathrm{C} 1$.

$\overline{3}$ Upstream and downstream mean with respect to the HERA lepton beam direction 


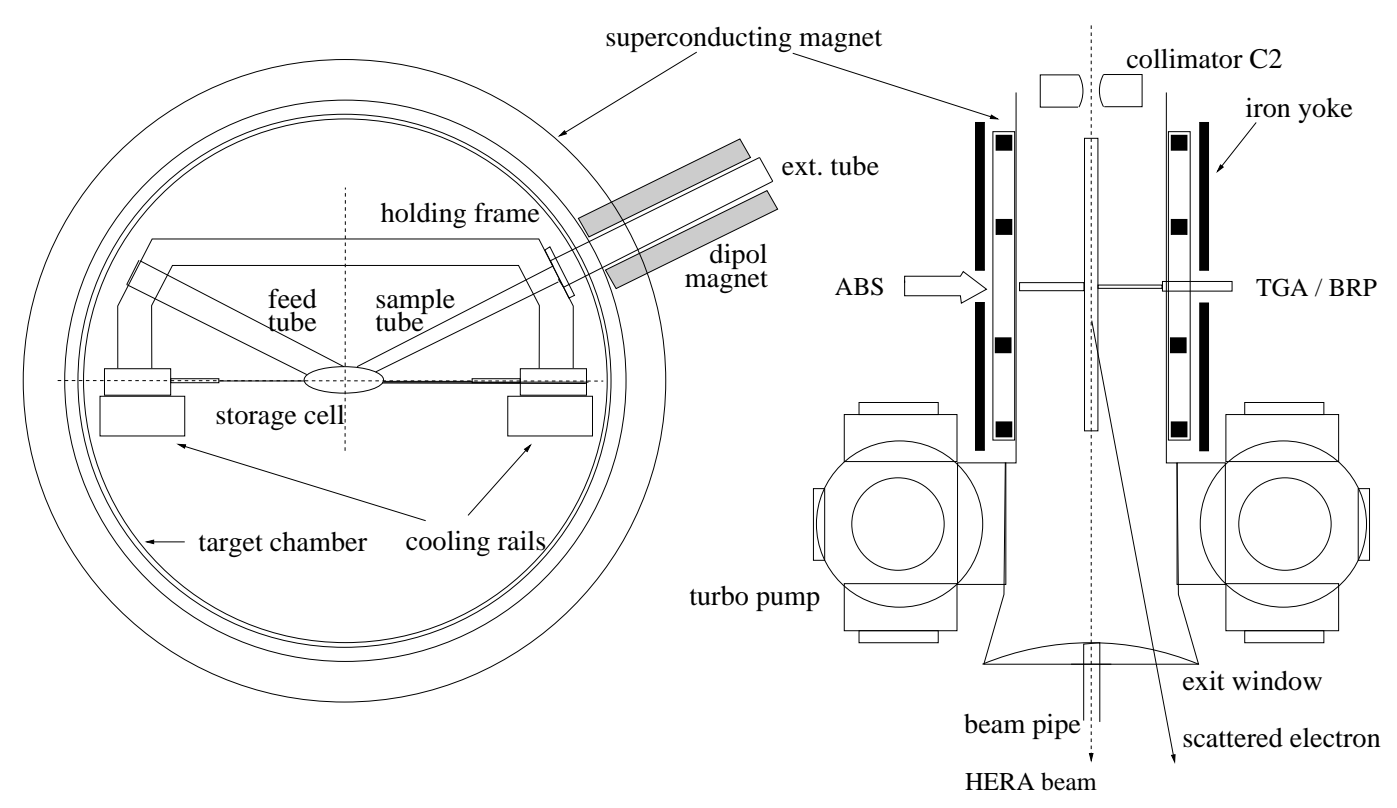

Fig. 2. Longitudinal target chamber and superconducting magnet viewed from downstream with respect to the HERA beam direction (left), and from above (right).

For longitudinal running the storage cell and vacuum chamber are immersed in a longitudinal field generated by a superconducting magnet. The magnet consists of four coils and an iron yoke designed to provide optimum field uniformity over the length of the storage cell while providing a central gap for the atomic beam to enter the cell. The magnetic field (up to $350 \mathrm{mT}$ ) provides the quantization axis for the spins of the polarized atoms in the storage cell, and decouples the spins of the nucleons and electrons. The passing HERA beam is bunched to provide high luminosity for the collider experiments H1 and ZEUS. As discussed in Sec. 4.2.4, this may induce resonant nuclear depolarization of the target gas. The induced resonances can be avoided by proper setting of the holding field, provided that its homogeneity is sufficient [13]. In the case of hydrogen, the maximum permissible variation of the magnetic field along the storage cell is $1.5 \%$. For operating with deuterium gas, this requirement was found to be less critical [16].

\subsection{Transverse magnet}

After the end of the longitudinal running in 2000, the HERMES target was modified for measurements with a hydrogen gas target in a transversely oriented magnetic holding field. The intensity of the transverse magnetic field was limited by the amount of synchrotron radiation power generated by the deflection of the beam by the target magnet ( $5 \mathrm{~kW}$ maximum), so it was decided to build a normal conducting magnet. As will be explained in Sec. 4.2.4, a better homogeneity of the transverse target magnet of $\Delta B \leq 0.15 \mathrm{mT}$ at a 

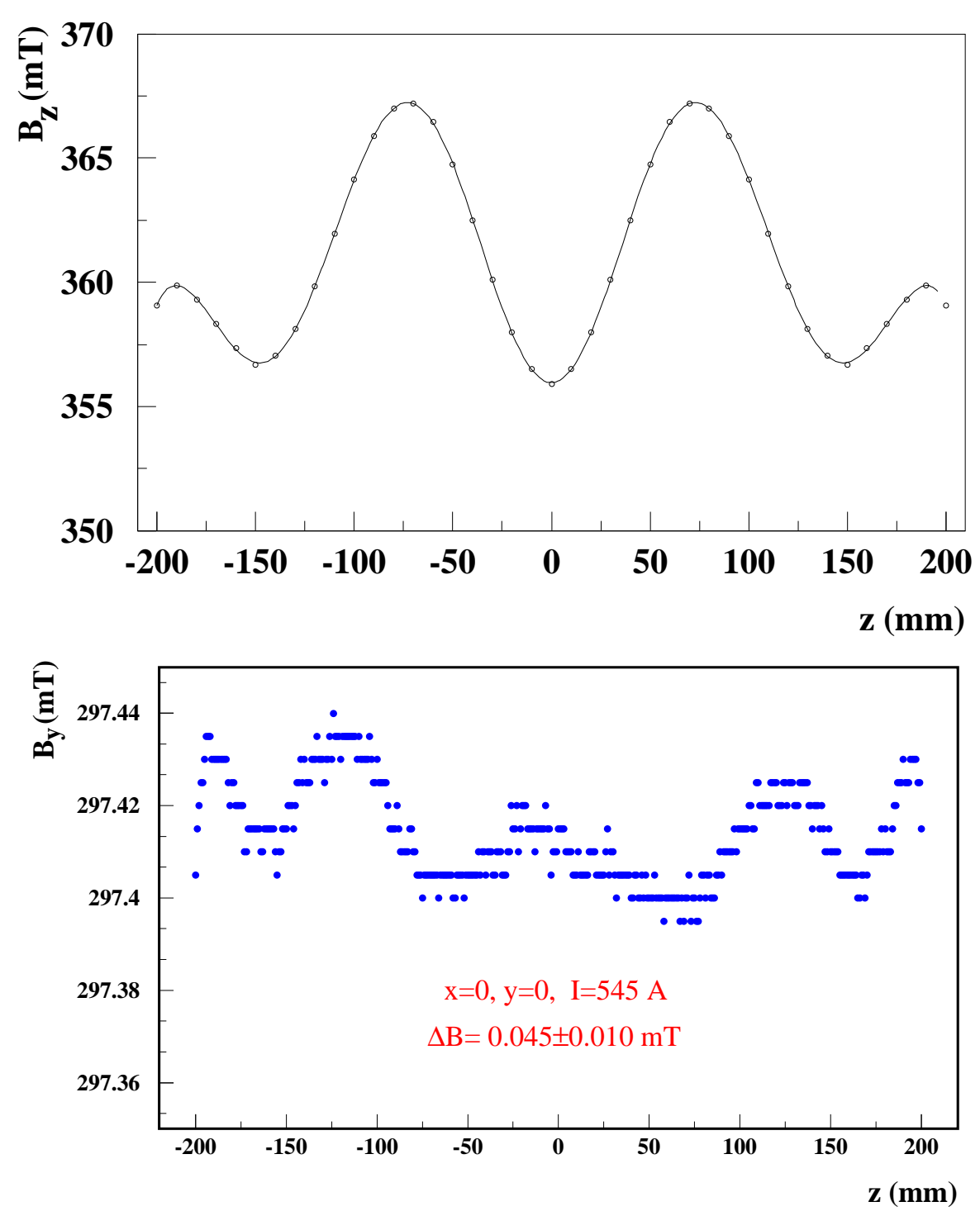

Fig. 3. Upper panel: field strength of the longitudinal target magnet measured along the HERA beam direction $z$, covering the full length of the storage cell. The line shows a polynomial fit to the measurement. Lower panel: transverse magnet field uniformity measured along the $z$ axis at the nominal field strength $B=297 \mathrm{mT}$. Deviations of $\Delta B_{y}=0.15 \mathrm{mT}$ (vert.) and $\Delta B_{x}=0.60 \mathrm{mT}$ (horiz.) have been measured within the cell volume.

field value of about $300 \mathrm{mT}$ was required.

The geometrical constraints imposed by the HERMES acceptance made it difficult to design a magnet fulfilling the uniformity requirement. After construction, the field uniformity was measured and improved by shimming the pole tips. At a field strength of $B=297 \mathrm{mT}$, maximum deviations of $\Delta B_{z}=$ $0.05 \mathrm{mT}, \Delta B_{y}=0.15 \mathrm{mT}$ and $\Delta B_{x}=0.60 \mathrm{mT}$ within the storage cell volume have been achieved, $z$ being the longitudinal direction, $y$ the vertical and $x$ the horizontal axis. The magnet was installed in July 2001. The strength of 
Table 1

\begin{tabular}{|c|c|c|}
\hline period of use & $\begin{array}{c}\text { cross section } \\
a \times b(\mathrm{~mm})\end{array}$ & $\begin{array}{c}\text { conductance } \\
(\mathrm{l} / \mathrm{s})\end{array}$ \\
\hline $05.96-11.99$ & $29.8 \times 9.8$ & $1.45 \sqrt{T / M}$ \\
\hline 01.99 (test) & $19.8 \times 8.0$ & $0.61 \sqrt{T / M}$ \\
\hline 12.99 - now & $21.0 \times 8.9$ & $0.80 \sqrt{T / M}$ \\
\hline
\end{tabular}

The history of cells used by HERMES. The lengths of the major and minor axes of the ellipse are indicated by $a$ and $b ; T$ is the temperature of the cell in $\mathrm{K}$, and $M$ is the atomic mass of the gas in amu.

the transverse field vs. $\mathrm{z}$ is shown in the lower plot of Fig. 3.

\subsection{The storage cell}

The HERMES storage cell [9] is made of two $75 \mu \mathrm{m}$ thin pure aluminum sheets, which are tightly spot-welded together. It is $400 \mathrm{~mm}$ long and has an elliptical cross-sectioned shape of $21 \times 8.9 \mathrm{~mm}^{2}$. All the aluminum parts of the cell are coated with Drifilm [17] to minimize gas depolarization and recombination caused by wall collisions $[18,19,20,21]$. A vented extension of the cell continues about $147 \mathrm{~mm}$ downstream of the cell before joining the thicker-walled wakefield-suppressing transition tube, to ensure that all scattered particles inside the spectrometer detector acceptance pass through the minimum thickness of material (see Fig. 4). The wake field suppressors are made of $100 \mu \mathrm{m}$ thick titanium, and confine the electromagnetic high frequency field of the bunched HERA beam, forming a gradual electrical transition between the storage cell and the HERA beam pipe.

Cells with three different elliptical cross sections have been used thus far. The mentioned size of $21 \times 8.9 \mathrm{~mm}^{2}$ is the one in use since December 1999, called the medium-sized cell. The largest was used until December 1999, and had a cross section of $29 \times 9.8 \mathrm{~mm}^{2}$. (A small one $\left(19 \times 8.0 \mathrm{~mm}^{2}\right)$ was tested in January 1999 and found to be too small.) Tab. 1 summarizes dimensions and conductances of the three cells that have been used. The conductance values apply to gas injected into the cell center.

The storage cell is mounted onto cooling rails, which are cooled by temperature regulated gaseous helium. The optimum operating temperature for hydrogen targets was found to be about $100 \mathrm{~K}$, where recombination and depolarization effects are low. Additionally, the lower conductance at this temperature leads to a higher target thickness by a factor $\sqrt{3}$ compared to room temperature. For deuterium gas the target temperature could be decreased further to about $65 \mathrm{~K}$, which increased the target thickness by about $23 \%$ compared to $100 \mathrm{~K}$. 


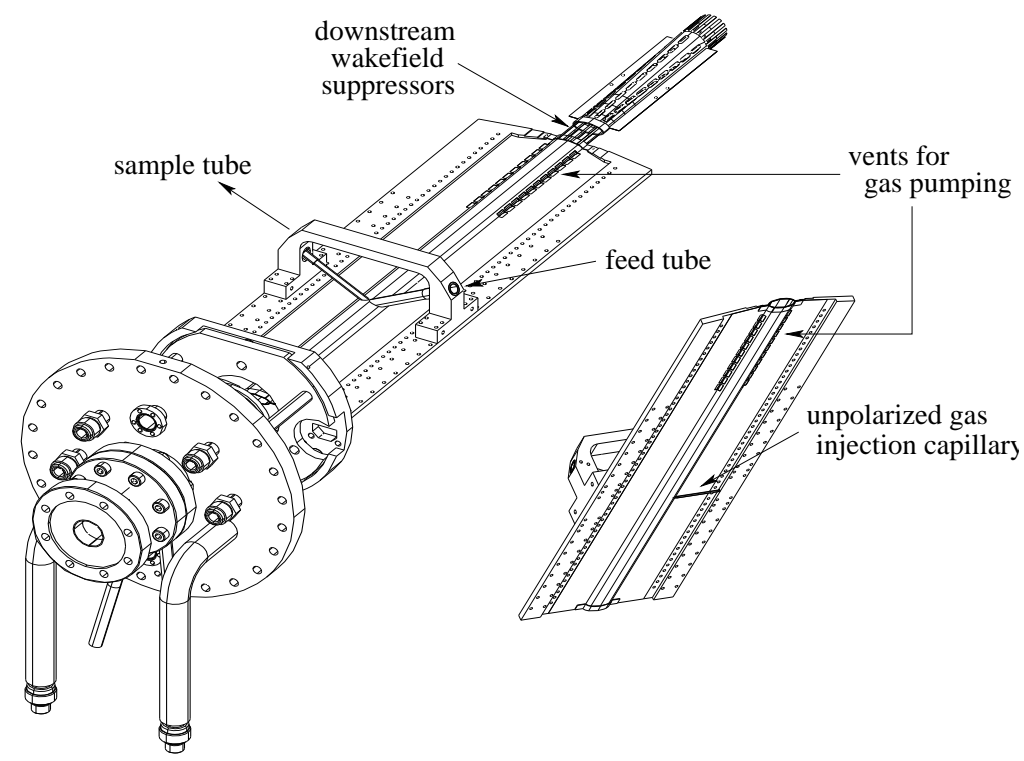

Fig. 4. The storage cell and its support flange.

The temperature of the cooling rails is monitored at three locations by platinum resistors. Since 1999 an additional thermocouple wire has been attached to the aluminum foil close to the cell ellipse to directly monitor the cell temperature during injection of the HERA beam when a temperature rise of about $5-10 \mathrm{~K}$ is usually detected. The cooling rails are mounted on the target chamber flange on the upstream end. Due to thermal contraction of the cell when being cooled, the position of the cell center changes relative to the atomic beam source and the Breit-Rabi polarimeter. From room temperature down to $100 \mathrm{~K}$ the cell center moves by $0.9 \mathrm{~mm}$. The alignment of the atomic beam source and Breit-Rabi polarimeter relative to the cell center includes an offset to compensate for this effect at the target operating temperature.

The cell is shown along with its support flange in Fig. 4. Two side tubes are connected to the beam tube, one to inject the polarized atoms and another smaller one to sample about $5 \%$ of the target gas and analyze it in the target gas analyzer and the Breit-Rabi polarimeter. The sample tube is tilted by $120^{\circ}$ with respect to the axis of the injection tube to ensure that the sampled atoms have thermalized with the storage cell wall. A permanent dipole magnet around the sampling tube outside the target chamber extends the magnetic holding field of the target magnet to prevent depolarization (see Fig. 2). A capillary near the location of the feed tube allows injection of unpolarized gas into the cell. 


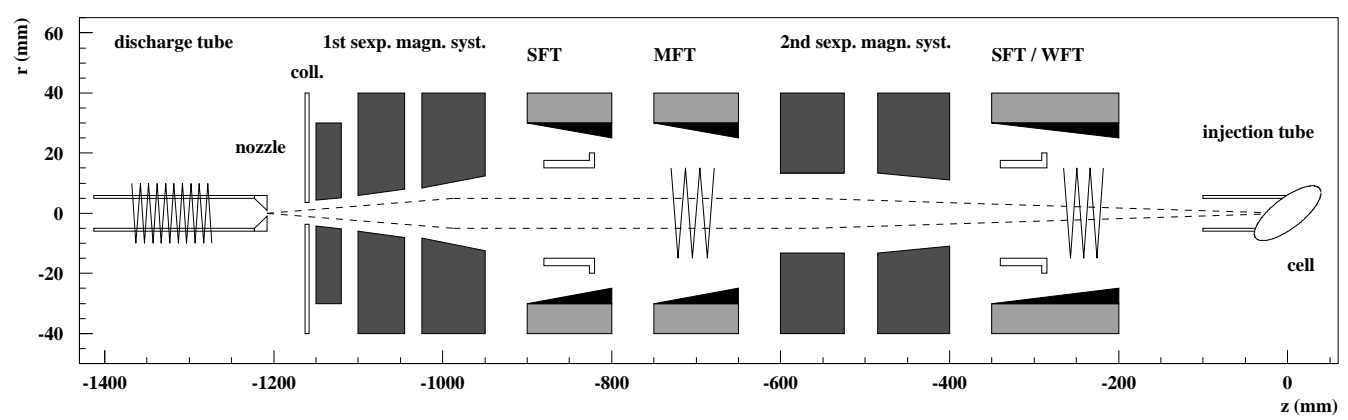

Fig. 5. Schematic view (from downstream of the electron beam) of the HERMES ABS with dissociator and collimator for beam formation. Two sets of sextupole magnets are located along the beam axis as are the high-frequency transitions. The axis of the $\mathrm{ABS}$ is tilted by $30^{\circ}$ downwards with respect to the horizontal plane.

\subsection{Atomic Beam Source}

The atomic beam source [8] consists of a dissociator, a powerful differential pumping system, a beam forming system, a sextupole magnet system to focus atoms with $m_{S}=+\frac{1}{2}$ into the storage cell, and adiabatic high-frequency transitions to manipulate the hyperfine population of the atomic beam. Injected fluxes of $\Phi^{A B S} \approx 6.5 \times 10^{16}$ atoms $/ \mathrm{s}$ in the case of hydrogen ( 2 states) and $\Phi^{A B S} \approx 4.5 \times 10^{16}$ atoms $/ \mathrm{s}$ in the case of deuterium (3 states) have been observed. These values are calculated from the density-dependent spin exchange relaxation [16]. The injected nuclear polarization $P_{z}^{i n j}$ was constantly above 0.97 for hydrogen and above 0.91 for deuterium. A schematic diagram of the ABS is depicted in Fig. 5.

Molecular hydrogen/deuterium gas enters the dissociator from the left. The molecules are dissociated by a radio frequency discharge with a frequency of $13.56 \mathrm{MHz}$ in a pyrex tube, producing a degree of dissociation up to $80 \%\left(\mathrm{H}_{2}\right.$, $\mathrm{D}_{2}$ ) at a flow of about $1 \mathrm{mbar}^{\mathrm{l}} \mathrm{s}^{-1}$ and a radio frequency power of $300 \mathrm{~W}$. To maximize the stability and the degree of dissociation, an amount of oxygen between 0.1 and 0.3 volume percent is added to the molecular gas.

A high degree of dissociation at higher flow can potentially be achieved using a recently developed microwave (MW) dissociator [22], which operates at a frequency of $2.45 \mathrm{GHz}$. With typical flows between 1 and $2 \mathrm{mbarls}^{-1}$ and a microwave power of about $600 \mathrm{~W}$, a degree of dissociation higher than $80 \%$ is achieved. The MW dissociator was used in the year 2000 when running with deuterium, when it provided a target thickness improvement of $5-10 \%$. The RF dissociator has been used since, because the ABS sextupole system is not optimized for the lower atomic velocity distribution produced by the MW dissociator, eliminating the possible benefit for hydrogen. 
The atomic gas flows through a conical nozzle with an opening diameter of $2 \mathrm{~mm}$, which is cooled to about $100 \mathrm{~K}$. The water produced in the discharge freezes in the nozzle. Although this beneficially reduces surface recombination, the ice layer continuously thickens and slowly reduces the gas conductance of the nozzle. After typically 3-5 days of operation, the pressure in the dissociator tube rises substantially, increasing volume recombination in the tube and resulting in a noticeable decrease in the degree of dissociation of gas exiting the nozzle. Full recovery is achieved by slightly warming up the nozzle to remove the ice layer.

After passing the cold nozzle, the gas expands into the vacuum of the dissociator chamber. A powerful differential pumping system with a total nominal pumping speed of more than $15000 \mathrm{ls}^{-1}$ suppresses scattering of the atomic beam, and ensures low gas flow into the HERA vacuum system.

The magnet system of the HERMES atomic beam source consists of a total of five Halbach type [23] segmented permanent sextupole magnets with a maximum poletip field of about 1.5 T. Every magnet consists of 24 segments of $\mathrm{Va}$ codym alloy ${ }^{4}$. Four high-frequency transition units are available: two between the sextupole magnets and two after the last magnet. The system provides enough flexibility that many different combinations of hyperfine states can be injected into the cell. Between the sextupoles, a strong field transition (SFT) and a medium field transition (MFT) can be used simultaneously, whereas the weak field transition (WFT) and the SFT after the sextupole magnets can only be used separately, because they share the coils for the static and gradient magnetic fields. The complete set of possible hyperfine populations that can be produced is shown in Tab. 2 .

\subsection{Target Gas Analyzer}

The target gas analyzer (TGA) [10] measures the atomic and molecular content of the gas extracted from the storage cell through the sample tube. The TGA arrangement, shown in Fig. 7, consists of a pair of baffles, a chopper, a $90^{\circ}$ off-axis quadrupole mass spectrometer (QMS) with a cross beam ionizer and a channel electron multiplier (CEM) for single ion detection. The TGA is integrated into the vacuum system of the BRP sextupole chamber, which is served by two cryopumps and a titanium sublimation pump, with a total pumping speed of about $7000 \mathrm{ls}^{-1}$. During operation, the pressure in the TGA detector is about $4 \cdot 10^{-9} \mathrm{mbar}$. Prior to normal operation, the TGA vacuum chamber is baked with temperatures up to $180^{\circ} \mathrm{C}$ for 48 hours. The BRP/TGA vacuum scheme is shown in Fig. 8.

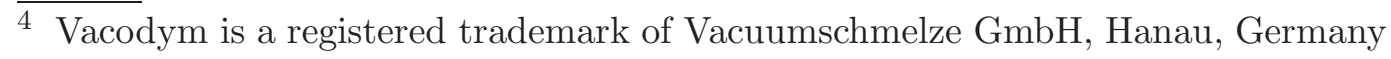




\begin{tabular}{|c|c|c|c|c|c|c|c|}
\hline Gas & HFT (betw. 6-poles) & HFT (after 6-poles) & Inj. States & $P_{e}$ & $P_{z}$ & $P_{z z}$ & Use \\
\hline \multirow{8}{*}{$\mathbf{H}$} & - & - & $|1\rangle,|2\rangle$ & +1 & 0 & - & $\mathrm{Cal}$ \\
\hline & - & WFT 1-3 & $|2\rangle,|3\rangle$ & 0 & -1 & - & Data \\
\hline & - & SFT 2-4 & $|1\rangle,|4\rangle$ & 0 & +1 & - & Data \\
\hline & SFT 2-4 / MFT 2-3 & - & $|1\rangle$ & +1 & +1 & - & Cal \\
\hline & WFT 1-3 / MFT 1-3 & - & $|2\rangle$ & +1 & -1 & - & Cal \\
\hline & SFT 2-4 / MFT 2-3 & WFT 1-3 & $|3\rangle$ & -1 & -1 & - & Cal \\
\hline & WFT 1-3 / MFT 1-3 & SFT 2-4 & $|4\rangle$ & -1 & +1 & - & Cal \\
\hline & WFT 1-3, SFT 2-4 & - & no state & - & - & - & Cal \\
\hline \multirow{11}{*}{$\mathrm{D}$} & - & - & $|1\rangle|2\rangle|3\rangle$ & +1 & 0 & 0 & Cal \\
\hline & SFT 2-5 & WFT 1-4 & $|3\rangle|4\rangle$ & 0 & -1 & +1 & Data \\
\hline & SFT 3-5 & SFT 2-6 & $|1\rangle|6\rangle$ & 0 & +1 & +1 & Data \\
\hline & MFT 1-4 & SFT 3-5 & $|2\rangle|5\rangle$ & 0 & 0 & -2 & Data \\
\hline & MFT 1-4 & SFT 2-6 & $|3\rangle|6\rangle$ & 0 & 0 & +1 & Data \\
\hline & MFT 3-4, SFT 2-6 & - & $|1\rangle$ & +1 & +1 & +1 & Cal \\
\hline & WFT $1-4$, SFT 2-6 & - & $|2\rangle$ & +1 & 0 & -2 & Cal \\
\hline & WFT 1-4, SFT 3-5 & - & $|3\rangle$ & +1 & -1 & +1 & Cal \\
\hline & MFT $3-4$, SFT 2-6 & WFT 1-4 & $|4\rangle$ & -1 & -1 & +1 & Cal \\
\hline & WFT $1-4$, SFT $3-5$ & SFT 3-5 & $|5\rangle$ & +1 & 0 & -2 & Cal \\
\hline & WFT 1-4, SFT 2-6 & SFT 2-6 & $|6\rangle$ & -1 & +1 & +1 & Cal \\
\hline
\end{tabular}

Table 2

Injection modes of the atomic beam source. The table shows the high frequency transition (HFT) units employed ( $2^{\text {nd }}$ and $3^{\text {rd }}$ columns), the hyperfine states injected in the target cell and the resulting electron $\left(P_{e}\right)$, nuclear $\left(P_{z}\right)$ and tensor $\left(P_{z z}\right)$ polarizations in the ideal case of $100 \%$ efficiency of the sextupole system and transition units, no depolarization inside the target cell and infinitely strong guide field. The last column clarifies the purpose of each mode (Cal: calibration, monitoring; Data: normal HERMES operation). The injection modes for hydrogen and deuterium single states are listed separately. For some modes the MFT between the sextupoles has to be tuned as a WFT 1-4. The no state injection mode is possible only for hydrogen and has been operational since April 2002. The adopted labeling for the hyperfine states is explained in the Breit-Rabi diagram of Fig. 6.

The TGA is mounted $7^{\circ}$ off-axis with respect to the BRP, in order to not interfere with the beam entering the polarimeter. The first baffle ensures that only gas from the sample beam can reach the detector, while the second one acts as a collimator, so that no atoms can hit the metal parts of the ionizer 

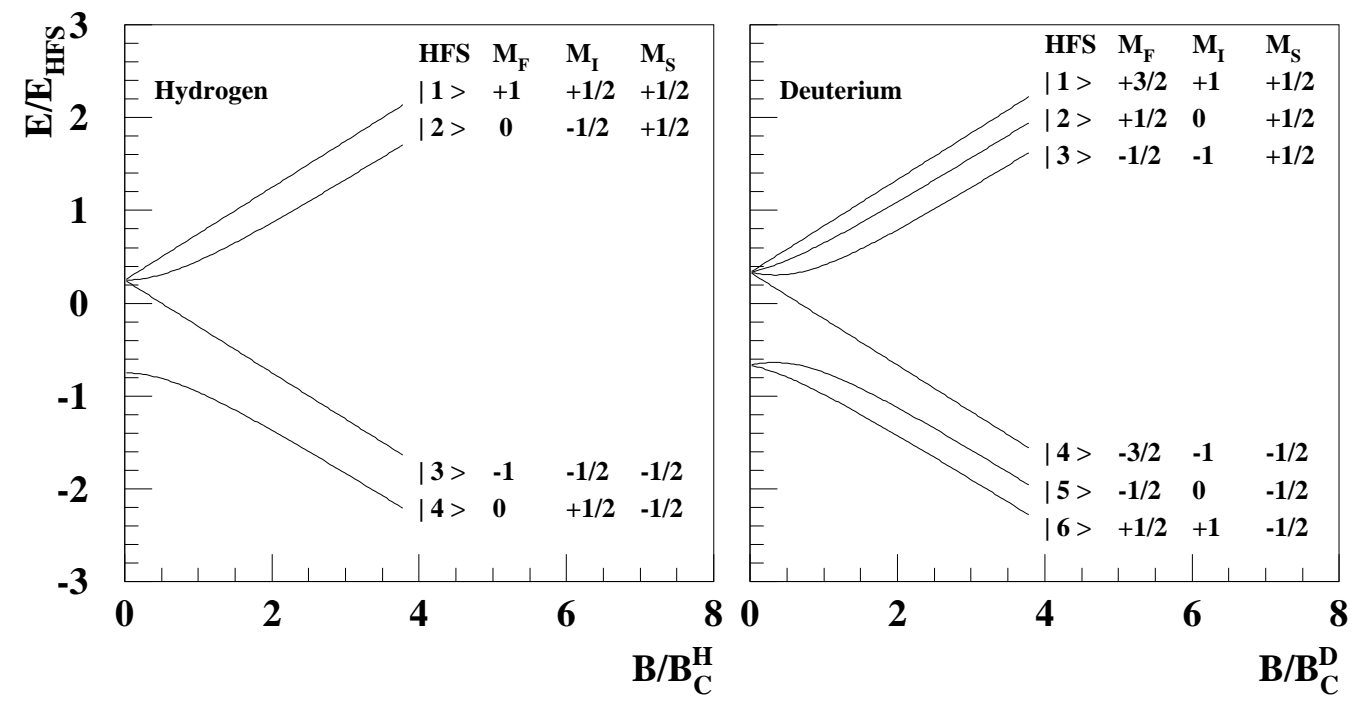

Fig. 6. Hyperfine energy levels of hydrogen (left) and deuterium (right) atoms as a function of the magnetic holding field (Breit-Rabi diagram) and corresponding labeling. The field values are scaled with the corresponding critical field and energy values with the corresponding hyperfine energy.

ABS
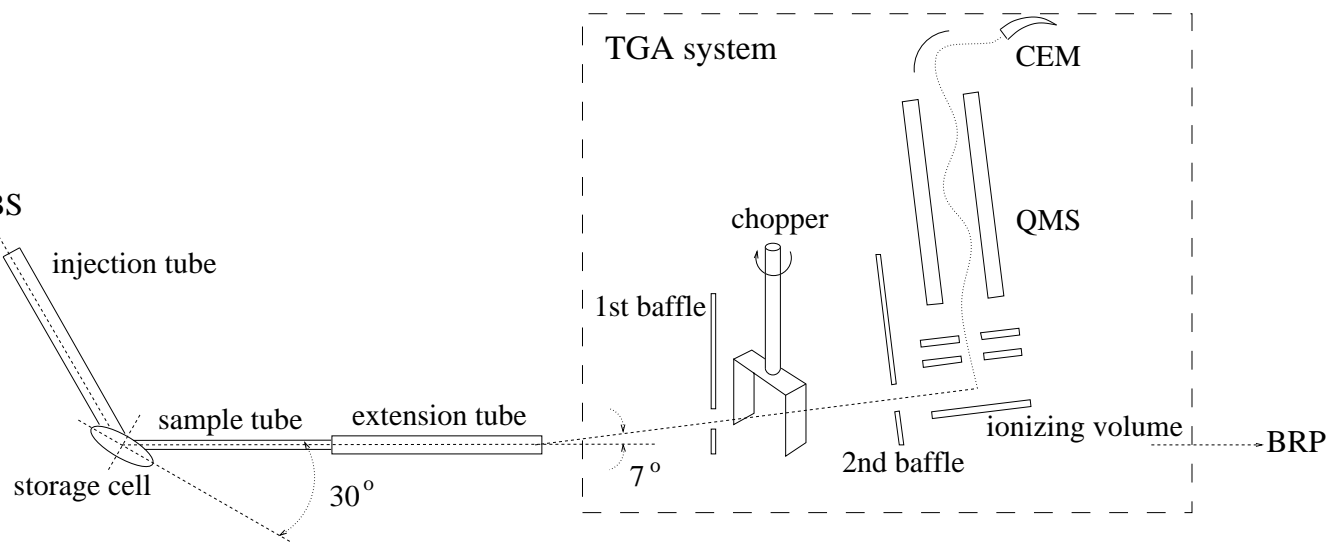

Fig. 7. A schematic diagram of the Target Gas Analyzer. The gas of atoms and molecules diffuses through the extension tube and is collimated by two baffles before entering the ionizing volume. The chopper is used for background subtraction

and recombine. The chopper, placed between the two baffles, rotates at a frequency of $5.5 \mathrm{~Hz}$, thus periodically blocking the sample beam in order to allow subtraction of the residual gas signal. Particles entering the detector are ionized by $70 \mathrm{eV}$ electrons, mass filtered with the QMS, and finally detected by the CEM. The pulses are counted by a 2048-bin time-resolving counter (TRC). The bin length is typically set to $175 \mu$ s so that two chopper turns are collected within the whole bin range as shown in Fig. 9. The synchronization between chopper status and counting rate is given by a trigger signal coming from the chopper motor. The beam rate is calculated by taking the difference between the number of counts collected with the chopper open and closed. 


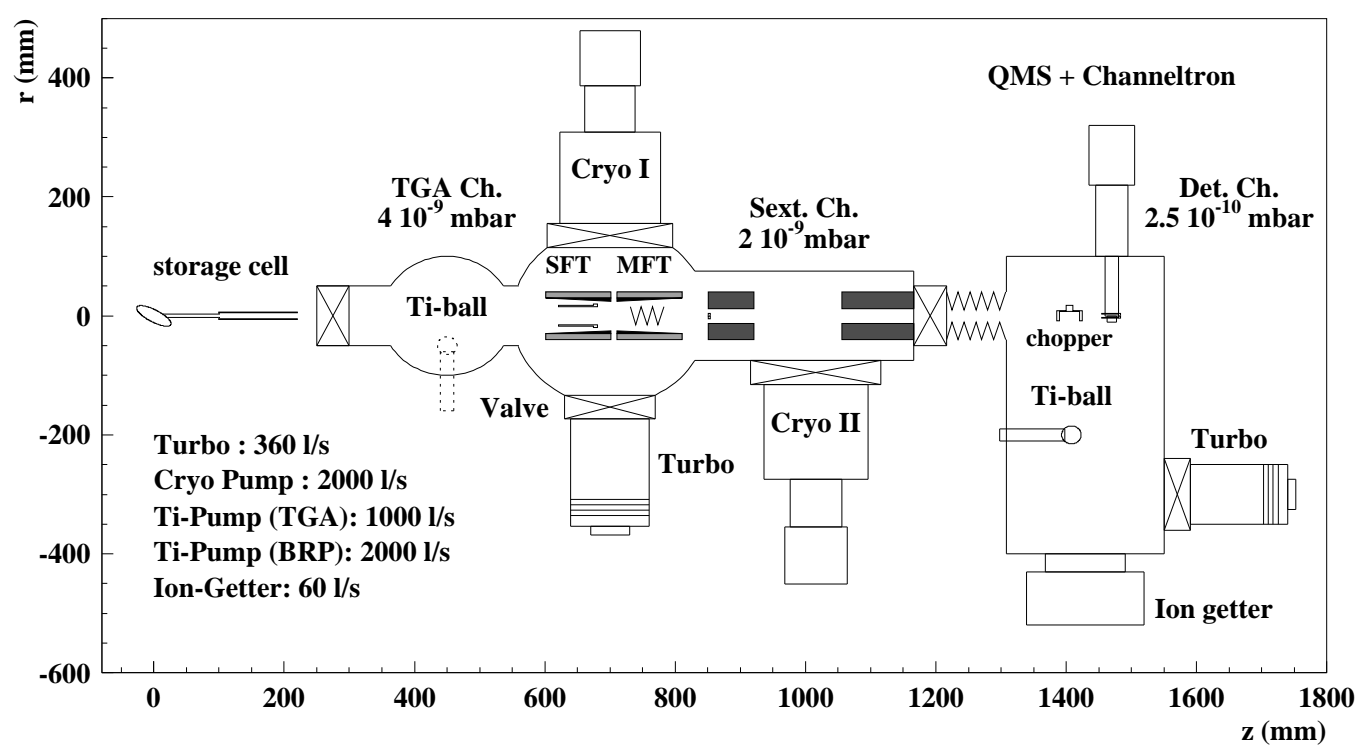

Fig. 8. Schematic view of the BRP/TGA vacuum system.

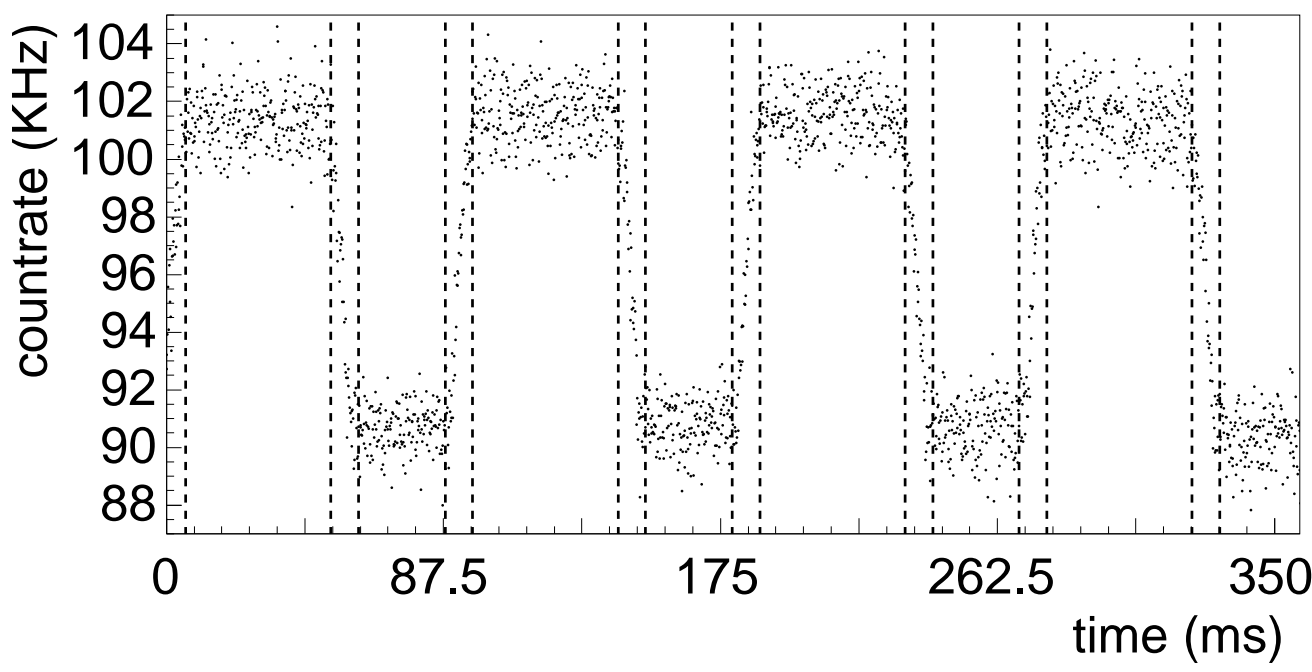

Fig. 9. Time spectrum of atomic count rates in the TGA. The vertical lines indicate the binning used to define the chopper positions open, undefined and closed.

\subsection{Breit-Rabi Polarimeter}

The Breit-Rabi polarimeter (BRP) [11] measures the relative populations $n_{i}$ of the hyperfine states of hydrogen (or deuterium) atoms contained in the sample beam. From this measurement, the absolute atomic polarizations can be calculated by applying the knowledge of the target magnetic field strength.

A schematic view of the BRP is shown in Fig. 10. From left to right, the 


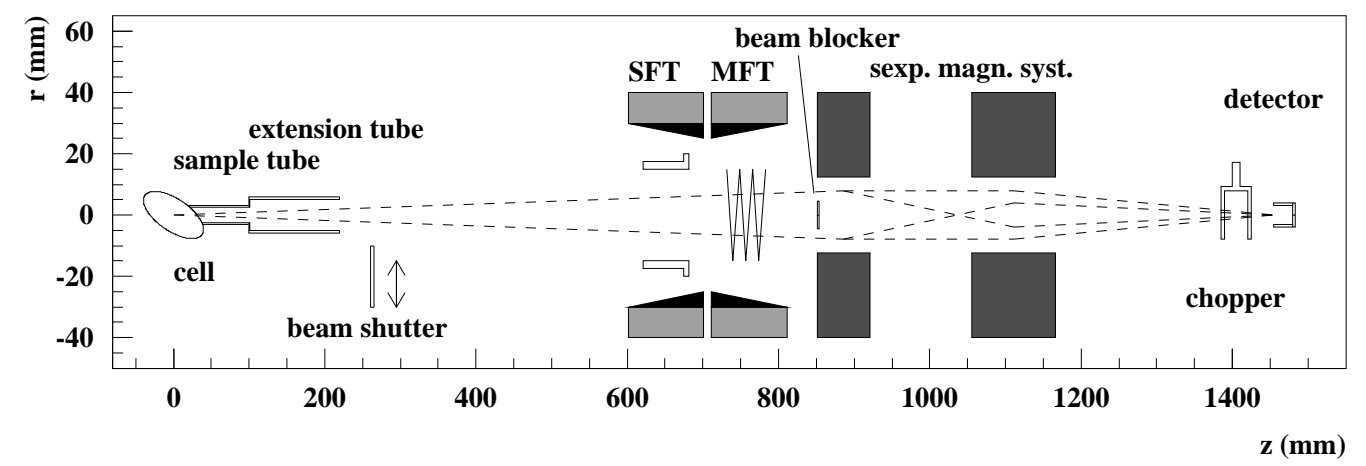

Fig. 10. Schematic layout of the BRP. The rf-transition units extend from $\mathrm{z}=600$ to $810 \mathrm{~mm}$. The beam blocker at the entrance to the first 6-pole magnet ensures $100 \%$ rejection of atoms with $m_{s}=-\frac{1}{2}$. The beam shutter is used to measure the hydrogen contribution coming from dissociative water ionization.

sample beam leaves the extension tube of the target cell, encountering first two hyperfine transition units, then a sextuple magnet system and eventually the detector stage. During operation, a differential pumping system keeps the pressure at $2 \times 10^{-9}$ mbar in the sextupole chamber and at $2.5 \times 10^{-10}$ mbar in the detector chamber.

Two transition units are used to exchange the populations between pairs of hyperfine states: a strong field transition unit (SFT) with tilted resonator that can be tuned for both $\pi$ and $\sigma$ transitions, and a medium field transition (MFT) unit that can induce various $\pi$ transitions according to the static field strength and gradient setting used. The sextupole system is composed of two magnets which focus the atoms of the sample beam with $m_{s}=+\frac{1}{2}$ towards the BRP geometrical axis and defocus the ones with $m_{s}=-\frac{1}{2}$. A $9 \mathrm{~mm}$ diameter beam blocker placed in front of the first sextupole magnet ensures that no atoms in $m_{s}=-\frac{1}{2}$ states can reach the detector. The sextupole system was redesigned in the year 2000 in order to improve the transmission probability at the typical cell temperature. The detector stage is identical to the one employed for the target gas analyzer: a cross beam ionizer, a quadrupole mass spectrometer (QMS) and a channel electron multiplier (CEM). In contrast to the TGA, only hydrogen (or deuterium) atoms are detected by the BRP. The chamber residual gas correction is carried out by using a chopper in the same way as described for the TGA. An additional beam shutter is placed just after the extension tube exit to measure the possible contribution of atomic hydrogen coming from the dissociation of $\mathrm{H}_{2} \mathrm{O}$ and $\mathrm{H}_{2}\left(\mathrm{D}_{2} \mathrm{O}, \mathrm{DHO}, \mathrm{D}_{2}\right.$ and $\mathrm{DH}$ in the deuterium case) present on the vacuum chamber walls ${ }^{5}$.

5 The beam shutter was installed at the beginning of the deuterium running period in 1998. During the 1996/'97 hydrogen running period, the contribution of dissociative hydrogen ionization was taken into account by detecting the intensity of masses 2 and $18 \mathrm{amu}$ in addition to mass $1 \mathrm{amu}[16]$. 


\section{Analysis of TGA and BRP signals}

\subsection{Interpretation of the TGA signals}

The measured TGA atomic (molecular) signal $S_{a}\left(S_{m}\right)$ is proportional to the product of the particle flux $\Phi_{a}\left(\Phi_{m}\right)$ into the TGA, the mean inverse velocity of the particles $\left\langle\frac{1}{v_{a}}\right\rangle\left(\left\langle\frac{1}{v_{m}}\right\rangle\right)$, the ionization cross section $\sigma_{a}^{i o n}\left(\sigma_{m}^{i o n}\right)$ and the detection probability $\epsilon_{a}\left(\epsilon_{m}\right)$. A dependence of the signal on the emission current is measured and taken into account by the normalization function $f\left(I_{\text {emiss }}\right)$. Taking all parameters into account one finds:

$$
S_{a, m} \propto f\left(I_{e m i s s}\right) \sigma_{a, m}^{i o n} \epsilon_{a, m}\left\langle\frac{1}{v_{a, m}}\right\rangle \Phi_{a, m} .
$$

The total flux $\Phi^{T G A}$ of $\mathrm{H}$ or D nucleons into the TGA can be related to the particle fluxes $\Phi_{a}$ and $\Phi_{m}$ by:

$$
\Phi^{T G A}=\Phi_{a}+2 \Phi_{m}
$$

The TGA geometrical acceptance is such that detected particles have bounced typically more than 100 times on the extension tube inner surface [16]. For this reason, particles entering the TGA detector have thermalized with the extension tube wall, which is a few tens of degrees $\mathrm{K}$ warmer than the cell. For thermalized atoms and molecules one has:

$$
\left\langle\frac{1}{v_{m}}\right\rangle=\sqrt{2}\left\langle\frac{1}{v_{a}}\right\rangle .
$$

Using eqs. (1), (2) and (3), it can be shown that the partial beam rates $S_{a}$ and $S_{m}$ can be combined to form the variable $S_{t o t}$ which is proportional to $\Phi^{T G A}$ :

$$
S_{t o t}=S_{a}+\kappa \sqrt{2} S_{m} \propto \Phi^{T G A}
$$

where $\kappa$ is the calibration constant of the TGA giving the relative sensitivity of the TGA for atomic and molecular beams:

$$
\kappa=\frac{\sigma_{a}^{i o n} \epsilon_{a}}{\sigma_{m}^{i o n} \epsilon_{m}} .
$$

Eq. (4) applies to the case of constant extension tube temperature $T_{\text {ext }}$ and emission current $I_{\text {emiss }}$. Taking into account the dependence of $S_{a}$ and $S_{m}$ on the measured ${ }^{6} T_{e x t}$ and $I_{\text {emiss }}$, one can define a temperature and emission normalized nucleon flow rate $\phi_{\text {tot }}$ into the TGA ionizer, which is proportional

\footnotetext{
${ }^{6}$ A thermocouple on the extension tube was first installed at the beginning of
} 
to the injected ABS intensity $\Phi^{A B S}$ (neglecting the small contribution from the target chamber rest gas):

$$
\phi_{t o t}=\frac{S_{t o t} \sqrt{T_{e x t}}}{f\left(I_{\text {emiss }}\right)} \propto \Phi^{A B S} .
$$

The quantity $\phi_{t o t}$ is a good monitor of ABS stability over time, as it is independent of recombination inside the storage cell. Following eq. (6) one can also express normalized nucleon flow rates for atoms, $\phi_{a}$, and for molecules, $\phi_{m}$, by:

$$
\begin{gathered}
\phi_{a}=\frac{S_{a} \sqrt{T_{e x t}}}{f\left(I_{\text {emiss }}\right)} \\
\phi_{m}=\frac{\sqrt{2} \kappa S_{m} \sqrt{T_{e x t}}}{f\left(I_{\text {emiss }}\right)} .
\end{gathered}
$$

The degree of dissociation of the sample beam, defined as the fraction of nucleons in atoms relative to all nucleons entering the TGA, is given by

$$
\alpha^{T G A} \equiv \frac{\phi_{a}}{\phi_{a}+\phi_{m}}=\frac{S_{a}}{S_{a}+\sqrt{2} \kappa S_{m}}
$$

The degree of dissociation $\alpha^{T G A}$, also called atomic fraction, is displayed by the TGA roughly once per minute.

\subsection{The calibration of the TGA}

The purpose of the TGA calibration is to determine the relative sensitivity $\kappa$ defined by eq. (5). The calibration can be performed by varying the relative amount of atoms and molecules at a constant injected ABS total flux:

$$
\phi_{\text {tot }}=\phi_{a}+\phi_{m}=\text { const }
$$

Using the relations (7) and (8), one can write

$$
\frac{\partial\left(\frac{S_{m} \sqrt{T_{e x t}}}{f\left(I_{\text {emiss }}\right)}\right)}{\partial\left(\frac{S_{a} \sqrt{T_{e x t}}}{f\left(I_{\text {emiss }}\right)}\right)}=-\frac{1}{\sqrt{2} \kappa},
$$

where the minus sign reflects the fact that an increase in $\phi_{m}$ must correspond to a decrease of $\phi_{a}$. Hence a fit of the normalized molecular signal $\phi_{m}$ as

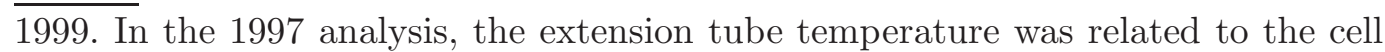
temperature using the expression $T_{\text {ext }}(T)=T_{0}+\frac{300-T_{0}}{300} T$, where $T_{0}$ is a constant estimated from calibration studies, and $\mathrm{T}$ is the measured cell temperature in $\mathrm{K}$. 
Table 3

\begin{tabular}{|c|c|}
\hline target/year & $\kappa$ \\
\hline \hline$H_{\|}(1997)$ & $0.551 \pm 0.026$ \\
\hline$D_{\|}(2000)$ & $0.861 \pm 0.024$ \\
\hline$H_{\perp}(2002)$ & $0.55 \pm 0.05$ \\
\hline
\end{tabular}

Summary of the values of TGA calibration constant $\kappa$ for the years 1997-2002.

function of the normalized atomic one $\phi_{a}$ results in a straight line with a slope of $-\frac{1}{\sqrt{2} \kappa}$.

Because the recombination process in the HERMES target is strongly temperature dependent, a controlled variation of the atomic fraction can be obtained by changing the temperature of the storage cell. A second possibility is to take advantage of an accidental beam loss in the region of the HERMES target which might temporarily change the surface property of the cell and cause a sharp drop of $\alpha^{T G A}$ followed by a slow recovery. The measured values for $\kappa$ are reported in Tab. 3.

A detailed description of the TGA calibration and its performance can be found in Ref. [10].

\subsection{Interpretation of the BRP signals}

The atomic signal detected by the BRP depends on the hyperfine population in the sample beam, the transmission probabilities of the sextupole system and on the setting of the transition units. For a stable storage cell temperature, the transmission of the magnetic system is fixed. The transition units can be switched on and off, changing the intensity of the beam reaching the detector. The BRP signal can be described by the following expression:

$$
S_{i}=c \sum_{a}\left(\sum_{b} \sigma_{b} T_{a b}^{i}\right) I_{a}
$$

where $I_{a}$ is the sample beam intensity (in atoms/s) of the atoms in hyperfine state $|a\rangle, T_{a b}^{i}$ describes the exchange of hyperfine states by the transition unit $^{7} i, \sigma_{b}$ is the sextupole transmission probability for state $|b\rangle$, and $c$ is a proportionality factor ${ }^{8}$. The goal of these measurements is to determine the four (six) sample beam intensities, $I_{a}$, for the hydrogen (deuterium) sample

7 If two units are working, the matrix $T^{i}$ has to be replaced by $T_{a b}^{i j}=\sum_{c} T_{a c}^{i} T_{c b}^{j}$

8 The expression (12) simplifies into $S_{o f f}=c \sum_{a} \sigma_{a} I_{a}$ in the case that no transition unit is switched on. 
beam. Summing eq. (12) over index $b$ one obtains:

$$
S_{i}=c \sum_{a} M_{i a} I_{a}
$$

where the measurement matrix $M_{i a}$ includes information concerning transmission probabilities and RF-transition efficiencies.

Assuming that $M_{i a}$ is known, one can solve the system of equations (13) for $I_{a}$ if, during a given ABS injection status, the BRP transition units are operated in at least four (six) different modes in the case of hydrogen (deuterium). The solution is given by

$$
I_{a}=c^{-1} \sum_{i} R_{a i} S_{i}
$$

where $^{9} R=M^{-1}$. The total sample beam intensity, $I_{t o t}$, is obtained by the taking the sum over all hyperfine states:

$$
I_{t o t}=\sum_{a} I_{a}
$$

Finally, the relative hyperfine populations $n_{a}$ are given by normalizing the intensities $I_{a}$ :

$$
n_{a}=\frac{I_{a}}{I_{t o t}}
$$

The polarizations of the atoms in the sample beam can now be calculated from the target magnetic field strength, as expressed by the following vectorial forms for hydrogen and deuterium, respectively:

$$
\begin{aligned}
\left(P_{e}, P_{z}\right)^{T} & =M_{P}^{H}\left(n_{1}, n_{2}, n_{3}, n_{4}\right)^{T} \\
\left(P_{e}, P_{z}, P_{z z}\right)^{T} & =M_{P}^{D}\left(n_{1}, n_{2}, n_{3}, n_{4}, n_{5}, n_{6}\right)^{T},
\end{aligned}
$$

where the matrices $M_{P}^{H}$ and $M_{P}^{D}$ contain the target holding field strength. e.g. for hydrogen:

$$
M_{P}^{H}=\left(\begin{array}{cccc}
-1 & a & 1 & -a \\
1 & -a & -1 & a
\end{array}\right),
$$

where $\mathrm{a}=\frac{k}{\sqrt{1+k^{2}}}, k=B / B_{c}, B_{c}=50.7 \mathrm{mT}$.

The statistical uncertainties of the measured signals $S_{i}$ are represented by the diagonal covariance matrix $C_{S}$. Hence, the covariance matrix $C_{I}$ of the intensities $I_{a}$ is given by:

$$
C_{I}=R C_{S} R^{T}=\left(M^{T} C_{S}^{-1} M\right)^{-1} .
$$

$\overline{9}$ If the number of collected signals is larger than the number of unknowns, one has to use the pseudo-inverse matrix $R=\left(M^{T} G_{S} M\right)^{-1} M^{T} G_{S}$, where $G_{S}$ is the inverse covariance matrix of the measured signals. This procedure is equivalent to a fit of the intensities $I_{a}$ to the signals $S_{i}$. 
The sum over all the elements of $C_{I}$ gives the statistical uncertainty $\Delta I_{\text {tot }}$ of the total intensity $I_{t o t}$ :

$$
\left(\Delta I_{t o t}\right)^{2}=\sum_{a b}\left(C_{I}\right)_{a b}
$$

The covariance matrix $C_{n}$ of the hyperfine populations $n_{a}$ is

$$
\left(C_{n}\right)_{a b}=\sum_{c d}\left(\frac{\partial n_{a}}{\partial I_{c}}\right)\left(C_{I}\right)_{c d}\left(\frac{\partial n_{b}}{\partial I_{d}}\right)
$$

while the covariance matrix of the polarization vector can be calculated in the following way:

$$
C_{P}=M_{P}^{H, D} C_{n}\left(M_{P}^{H, D}\right)^{T} .
$$

From $C_{P}$ one can extract the statistical uncertainty of the polarization measurement.

During normal running conditions, the entire automated procedure to measure and calculate the polarization vectors and their uncertainties took roughly $60 \mathrm{~s}$ (90 s) for hydrogen (deuterium), resulting in a statistical uncertainty of about \pm 0.013 for hydrogen. For deuterium the statistical uncertainty was about \pm 0.031 for positive and \pm 0.022 for negative nuclear polarization. After an optimization of the BRP sextupole system, and an increase in the diameter of the sample tube, the statistical uncertainty of a 60 s measurement for hydrogen was reduced to less than $0.5 \%$.

\subsection{The BRP calibration}

In the previous section it was shown that the atomic polarizations can be calculated from the measured BRP signals assuming the knowledge of the measurement matrix $M_{i a}$. This includes the efficiencies of the transition units and the transmission probabilities of the sextupole system for various hyperfine states. The procedure used to determine these parameters is called the BRP calibration.

The basic idea of the calibration measurement is to make the system of equations (13) overdetermined so that additional parameters like efficiencies or sextuple transmissions can be extracted through a fit to the data. Due to the fact that the transition units can be combined in several possible ways, the number of signals which can be collected during a polarization measurement exceeds the number of hyperfine states $N_{h f s}$. However, the number of unknowns that can be determined in this way is still less than the total number of parameters. The problem can be overcome by cycling this measurement through several (total number $N_{A B S}$ ) injection modes (see Tab. 2) so that an adequate number of signals can be collected. 


\begin{tabular}{|c|c|c|c|c|c|}
\hline BRP-Signal & Name & MFT-RF & MFT & SFT-RF & SFT \\
\hline \hline OFF & off & OFF & OFF & OFF & OFF \\
\hline SFT 1-4 & s14 & OFF & OFF & ON & $1-4$ \\
SFT 2-4 & $\mathrm{s} 24$ & OFF & OFF & ON & $2-4$ \\
\hline MFT 1-3 & m13c14 & ON & $1-3$ & OFF & $1-4$ \\
MFT 1-3 & m13c24 & ON & $1-3$ & OFF & $2-4$ \\
MFT 2-3 & m23c14 & ON & $2-3$ & OFF & $1-4$ \\
MFT 2-3 & $\mathrm{m} 23 \mathrm{c} 24$ & ON & $2-3$ & OFF & $2-4$ \\
\hline MFT 1-3/SFT 1-4 & $\mathrm{m} 13 \mathrm{~s} 14$ & ON & $1-3$ & ON & $1-4$ \\
MFT 1-3/SFT 2-4 & $\mathrm{m} 13 \mathrm{~s} 24$ & ON & $1-3$ & ON & $2-4$ \\
MFT 2-3/SFT 1-4 & $\mathrm{m} 23 \mathrm{~s} 14$ & ON & $2-3$ & ON & $1-4$ \\
MFT 2-3/SFT 2-4 & $\mathrm{m} 23 \mathrm{~s} 24$ & ON & $2-3$ & ON & $2-4$ \\
\hline
\end{tabular}

Table 4

Possible switching modes of the BRP transition units for hydrogen running. During a calibration measurement all signals are collected, while for a polarization measurement only a subset of them is chosen.

\subsubsection{The transition efficiencies}

We first discuss the method to determine the transition efficiencies, assuming a priori knowledge of the sextupole transmissions $\sigma_{n}$ for each hyperfine state. Calibration of those transmissions will then be discussed in Sec. 3.4.2.

The BRP contains two transition units, a strong field (SFT) and a medium field (MFT) unit. The SFT can drive two transitions for hydrogen $(|1\rangle \leftrightarrow|4\rangle$ and $|2\rangle \leftrightarrow|4\rangle$ ) and five for deuterium. The efficiencies of the SFT transitions in the case of hydrogen are called $\varepsilon_{s 14}$ and $\varepsilon_{s 24}$. The MFT is a multi-step transition unit which can drive two sub-transitions for hydrogen $(|1\rangle \leftrightarrow|2\rangle$ and $|2\rangle \leftrightarrow|3\rangle^{10}$ ) and three for deuterium. For hydrogen the MFT is tuned either as a $|1\rangle \leftrightarrow|3\rangle$ (which is achieved by applying the $|1\rangle \leftrightarrow|2\rangle$ and $|2\rangle \leftrightarrow|3\rangle$ transitions sequentially) or as a $|2\rangle \leftrightarrow|3\rangle$ only. In the latter case the MFT unit is tuned to avoid the $|1\rangle \leftrightarrow|2\rangle$ sub-transition. Although nearly zero, the residual $|1\rangle \leftrightarrow|2\rangle$ efficiency for the $|2\rangle \leftrightarrow|3\rangle$ tune of the MFT has to be taken into account.

Due to the limited space available for the BRP equipment, there is cross-talk between the two BRP transition units when both static fields are switched

$\overline{10}$ The order of the sub-transitions depends on the sign of the gradient field, which is negative in the HERMES BRP [16]. 
on, so that the tuning of the MFT is influenced by the SFT field, whereas the SFT is not influenced by the (low) MFT field. The MFT efficiencies have to be measured separately for each SFT setting, as they depend on the exact static field. For hydrogen this leads to an increase in the number of MFT efficiencies from four to eight. The various MFT efficiencies are identified by means of the subscripts $c 14$ (for SFT 1-4 static field on) or $c 24$ (SFT 2-4 static field on) and by the subscripts $\mathrm{r} 13$ or $\mathrm{r} 23$ which indicate the particular sub-transition being considered. For example, the notation $\varepsilon_{m 13 r 23 c 24}$ represents the efficiency of the transition MFT 1-3, step 2-3, tuned together with the static field of the SFT 2-4. The total number of efficiencies $N_{\text {eff }}$ is 10 for hydrogen and 41 in the case of deuterium. The number $N_{B R P}$ of possible BRP signals related to various transition states is 11 for hydrogen (as shown in Tab. 4) and 29 for deuterium.

If the ABS is operated in $N_{A B S}$ different injection modes, and the BRP transition units switch through all possible $N_{B R P}$ states, the total number of collected signals is $N_{s}=N_{A B S} N_{B R P}$. In this way, recalling eq. (13), one obtains a system of $N_{s}$ equations containing $N_{u}=N_{\text {eff }}+N_{A B S} N_{h f s}$ unknowns ${ }^{11}$, where $N_{h f s}=4(6)$ in the case of hydrogen (deuterium). The system can be solved if $N_{f}=N_{s}-N_{u} \geq 0$, where $N_{f}$ counts the degrees of freedom and is given by

$$
\begin{aligned}
N_{f} & =N_{s}-N_{u} \\
& =\left(N_{B R P}-N_{h f s}\right) N_{A B S}-N_{e f f} .
\end{aligned}
$$

In such a case, both the hyperfine state intensities $I_{a}$ and the transition efficiencies $\varepsilon$ can be determined. For hydrogen, the calibration measurement makes use of $7 \mathrm{ABS}$ injection modes, resulting in $N_{f}=39$. In the case of deuterium two possible options have been developed, one with 5 ABS modes $\left(N_{f}=74\right)$ and the other with $6\left(N_{f}=97\right)$.

The solution of the system is carried out by applying a Runge-Kutta method as explained in [11]. The resulting covariance matrix $C_{x}$ has the form

$$
C_{x}=\left(\begin{array}{cc}
C_{\varepsilon} & C_{\varepsilon, I} \\
C_{I, \varepsilon} & C_{I}
\end{array}\right),
$$

where $C_{\varepsilon}$ contains uncertainties and covariances of the efficiencies and is used to calculate the systematic uncertainty of the polarization measurement. The resulting systematic uncertainty induced by the determination of the transition efficiencies of the order of $1.5 \%$. Tab. 5 summarizes the transition effi-

\footnotetext{
${ }^{11}$ During the evaluation of the transition efficiencies, the sextuple system transmissions probabilities are assumed to be constant.
} 


\begin{tabular}{|c|c|c|c|c|}
\hline Name & MFT & SFT & Efficiency (\%) & uncertainty (\%) \\
\hline \hline$\varepsilon_{s 14}$ & - & $1-4$ & 99.3 & 1.34 \\
$\varepsilon_{s 24}$ & - & $2-4$ & 101.0 & 1.03 \\
\hline$\varepsilon_{m 13 r 12 c 14}$ & $1-3$ & $1-4$ & 101.0 & 1.03 \\
$\varepsilon_{m 13 r 23 c 14}$ & & & 98.6 & 1.41 \\
\hline$\varepsilon_{m 23 r 12 c 14}$ & $2-3$ & $1-4$ & -1.0 & 1.13 \\
$\varepsilon_{m 23 r 23 c 14}$ & & & 94.9 & 1.23 \\
\hline$\varepsilon_{m 13 r 12 c 24}$ & $1-3$ & $2-4$ & 99.9 & 0.42 \\
$\varepsilon_{m 13 r 23 c 24}$ & & & 90.3 & 0.82 \\
\hline$\varepsilon_{m 23 r 12 c 24}$ & $2-3$ & $2-4$ & 0.0 & 1.10 \\
$\varepsilon_{m 23 r 23 c 24}$ & & & 97.4 & 1.04 \\
\hline
\end{tabular}

Table 5

BRP transition unit efficiencies measured during the 1997 hydrogen running fixing the sextupole transmission ratio to $r_{21}=1.029$. Each efficiency is shown along with an explanation of the MFT and SFT static field setting.

ciency measurements performed in 1997 with hydrogen. The efficiencies of the transitions for deuterium running can be found in Ref. [11].

\subsubsection{The sextupole transmissions}

In the previous section it was shown how the efficiencies $\varepsilon_{i}$ of the BRP transition can be measured, assuming a priori knowledge of the sextupole transmissions $\sigma_{n}$ for each hyperfine state. The attempt to fit both $\varepsilon_{i}$ and all of the $\sigma_{n}$ within the same algorithm fails. Nevertheless, the transmission probabilities can be determined by separating the fitting procedure into two steps, as shown below.

In the BRP (Fig. 10), atoms with $m_{S}=-\frac{1}{2}$ are either defocused or stopped by the beam blocker, having therefore zero transmission probability $\left(\sigma_{3}=\right.$ $\sigma_{4}=0$ for hydrogen and $\sigma_{4}=\sigma_{5}=\sigma_{6}=0$ for deuterium). Moreover, due to the normalization (eq. (16)), only ratios of transmission probabilities are required for measuring the hyperfine populations. Thus the remaining required parameters are $\sigma_{2} / \sigma_{1}$ for hydrogen and $\sigma_{2} / \sigma_{1}$ and $\sigma_{3} / \sigma_{1}$ for deuterium.

The Stern-Gerlach force of the sextupole magnets is weaker for hydrogen atoms in state $|2\rangle$ compared to state $|1\rangle$ for magnetic fields $B$ of the order or less than the hydrogen critical field $B_{c}=50.7 \mathrm{mT}$, near the axis of the sextupole system. Thus near-axis atoms in hyperfine states $|1\rangle$ and $|2\rangle$ will experience a different transmission efficiency and the transmission ratio $r_{12}=\sigma_{1} / \sigma_{2}$ is 
expected to exceed unity by a small amount. Due to the smaller hyperfine coupling of deuterium, $B_{c}=11.7 \mathrm{mT}$, the deviation from unity of the ratio $\sigma_{2} / \sigma_{1}$ and $\sigma_{3} / \sigma_{4}$, is expected to be significantly smaller than for hydrogen. Ray tracing simulations [8] show that the deviation is in fact negligible. From these consideration it follows that the only transmission ratio that needs to be measured is $r_{21}=\sigma_{2} / \sigma_{1}$ for hydrogen.

From the BRP calibration the transition efficiencies are extracted as a function of the parameter $r_{21}$. The transmission ratio can thus be evaluated by minimizing the $\chi^{2}$ of the calibration results. As the transmission probability of the sextupole system depends on the velocity distribution of the atomic sample, the storage cell temperature $T_{\text {cell }}$ has to stay constant over the calibration measurement. This procedure applied to a calibration measurement taken at $T_{\text {cell }}=95 \mathrm{~K}$ has produced the following result [11]:

$$
\left.\frac{\sigma_{2}}{\sigma_{1}}\right|_{95 K}=1.029 \pm 0.0015 .
$$

The small uncertainty of the measured ratio can be neglected in the calculation of the systematic uncertainty of the polarization measurement. The potential cell temperature dependence of the transition efficiencies can be neglected as the velocity distribution of the sample is fixed by the BRP sextupole system. Therefore, a polarization measurement performed collecting at least one signal more than the minimum required number (as is always the case) can be used to fit the ratio $r_{21}$ for various cell temperatures.

A more detailed description of the BRP calibration and performance can be found in Ref. [11].

\section{Processes occuring inside the Storage Cell}

Inside the storage cell, the atoms of the target may experience several interactions that have an influence on the average target polarization $P_{T}$. Their complete understanding is therefore fundamental for the reduction of the systematic uncertainty of the target polarization. In this section an overview of these processes will be given, while a quantitative estimate of their contributions for the various running periods of the HERMES target can be found in Sec. 6. The processes are divided into two categories - recombination processes and spin relaxation processes.

Recombination and relaxation by wall collisions are surface effects whereas spin exchange collisions and resonant interaction of beam and target gas happen in the gas storage cell volume. The operating conditions of the target are chosen such that these unwanted effects are suppressed as much as possible. It 
should be noted that spin relaxation can be studied and monitored with good statistics by means of the BRP, whereas the residual polarization after recombination can be studied only using a scattering process like deeply inelastic scattering with a much lower rate.

\subsection{Recombination Processes}

The atoms that are injected into the HERMES storage cell may recombine to molecules, possibly keeping part of their nuclear polarization. Recombination [12] can in principle take place either on the storage cell walls (surface recombination) or in the gas phase (volume recombination). At the low gas densities of the HERMES target, volume recombination is negligible. On the cell walls two types of recombination processes with surface catalysis can be distinguished, which are expected to result in different residual nuclear polarization in the molecules:

- the Langmuir-Hinselwood (L-H) process, where two adsorbed thermalized atoms react with each other;

- the Eley-Rideal (E-R) process, where an atom from the gas phase reacts directly with an adsorbed atom [25].

The residual polarization of the surface atoms recombining through the E-R mechanism in the cell was measured with the HERMES spectrometer, making use of a well known DIS asymmetry. The result was presented in Ref. [14] and will be discussed in section 5.2.

Since the storage cell temperature is usually kept below $140 \mathrm{~K}$, a small amount of water originating from the ABS dissociator freezes on the surface and grows into an ice layer on top of the Drifilm surface. The presence of water is important since water is known to inhibit hydrogen or deuterium recombination. Fresh storage cell coatings are hydrophobic, but secondary particles from the HERA beam quickly change the chemical structure of the Drifilm surface, causing the surface to become less hydrophobic. This was predicted to occur in the longitudinal holding field by simulations of acceleration by the beam bunch electric fields of positive ions produced by the beam in the target gas. Even when not at cyclotron resonance, the ions stochastically gain energy until they hit the cell wall with energies of order $100 \mathrm{eV}$. Surface doses in the range $10 \mathrm{~Gy} / \mathrm{s}$ can occur, depending on the magnetic holding field and the beam current. Furthermore, the downstream portion of the small cell used since December 1999 is exposed to synchrotron radiation that is single-scattered from collimator C1. Fortunately, it has been observed that during normal operation, the quality of the cell surface gradually recovers and the amount of recombination in the cell decreases, thanks to the steady accumulation of ice on the 
surface.

A detailed description of the recombination studies performed on the HERMES storage cell can be found in Ref. [12].

\subsection{Spin relaxation}

Three different atomic spin relaxation mechanisms can in principle be observed to occur in the HERMES target, as they cause a change in the hyperfine population inside the storage cell. One relaxation process takes place on the wall of the storage cell. Another is due to two-body spin-exchange collisions of atoms in the gas phase. In the third mechanism the high frequency fields generated by the passage of the very short HERA beam bunches can cause a depolarization in the target atoms under certain conditions [13]. Spin relaxation of atomic hydrogen by wall or spin exchange collisions with various types of wall coatings has been under study for many years in the context of a hydrogen maser for low holding fields [6]. For the HERMES target further studies of the magnetic field dependence of spin relaxation as well as studies of the temperature dependence and density dependence of the transition spectra have been carried out $[16,30,31,32,33]$. The results of these studies are summarized in the following sections.

\subsubsection{Wall relaxation}

The atoms injected by the atomic beam source experience several hundred wall collisions during their diffusion process through the storage cell. An attractive Van-der-Waals potential between the storage cell wall and a diffusing atom causes the atom to be physisorbed, i.e. to stay for a certain time close to the surface until it is desorbed again. The mean residence time on the wall $\tau_{s}$ is described by the Arrhenius equation:

$$
\tau_{s}=\tau_{0} e^{\left(\frac{E_{b}}{k T}\right)}
$$

where $\tau_{0}$ is the high temperature limit of $\tau_{s}, E_{b}$ and $T$ are the adsorption energy and the temperature respectively, and $k$ is the Boltzmann constant. During the time an adsorbed atom stays on the wall $\left(\approx 10^{-10} \mathrm{~s}\right.$ for the HERMES cell), the magnetic moment of its unpaired electron interacts with magnetic moments on the surface by means of a dipolar coupling and/or with other unpaired electrons through exchange interactions [18,19,20,21]. Depending on the surface type, temperature and magnetic field, one can define a probability that the atom leaves the wall in a certain hyperfine state. This is described by means of transition probabilities $W_{j k}$, each of which represents the probability 
to find an atom in state $|k\rangle$ that was in state $|j\rangle$ prior to the wall collision. The theoretical description and the calculation of the transition probability matrix for the HERMES target can be found in [16].

\subsubsection{Spin Exchange Depolarization}

Atoms colliding with each other in the gas phase can undergo spin exchange, where hyperfine transitions can occur via interaction between the electron spins [34]. Due to the conservation of angular momentum, the sum of the magnetic quantum numbers $m_{F}$ before and after the spin exchange collision has to be the same:

$$
\Delta \sum_{i} m_{F}=0
$$

One can see that if an initial population of the gas consists only of pure state $|1\rangle$ or $|3\rangle$ in the case of hydrogen and $|1\rangle$ or $|4\rangle$ in the case of deuterium, no relaxation by means of spin exchange is possible. This is actually not the case for the HERMES cell, where to double the density, more than one atomic state is injected during normal operation. The effect of spin exchange collisions on the hyperfine populations can be described by a tensor $M_{j k}^{i}$, which gives the probability to find an atom in state $|i\rangle$ after a collision of two atoms in the state $|j\rangle$ and $|k\rangle$ prior to the collision: The number of spin exchange collisions per unit time and volume is

$$
\dot{N}_{s e}=n^{2} \sigma_{s e}\left\langle v_{r}\right\rangle
$$

where $n$ is the gas density, $\sigma_{s e}$ is the spin exchange cross section and $v_{r}$ is the relative velocity of the atoms. The density dependence of spin-exchange collision relaxation, combined with the knowledge of $\sigma_{s e}[35,36]$, was used to determine the density inside the HERMES cell [16]. The results will be reported in Sec. 6 .

\subsubsection{Evolution equation for the description of the spin relaxation process}

The various spin relaxations due to wall collisions and spin exchange collisions cannot be treated separately. After an interaction with the surface during a wall collision, an atom in the gaseous phase can then interact through spin exchange collision and, at the next wall collision, interact again with the surface. Since the atoms are constantly exposed to both interactions, an appropriate description is given by an evolution equation for the hypefine fractional pop- 

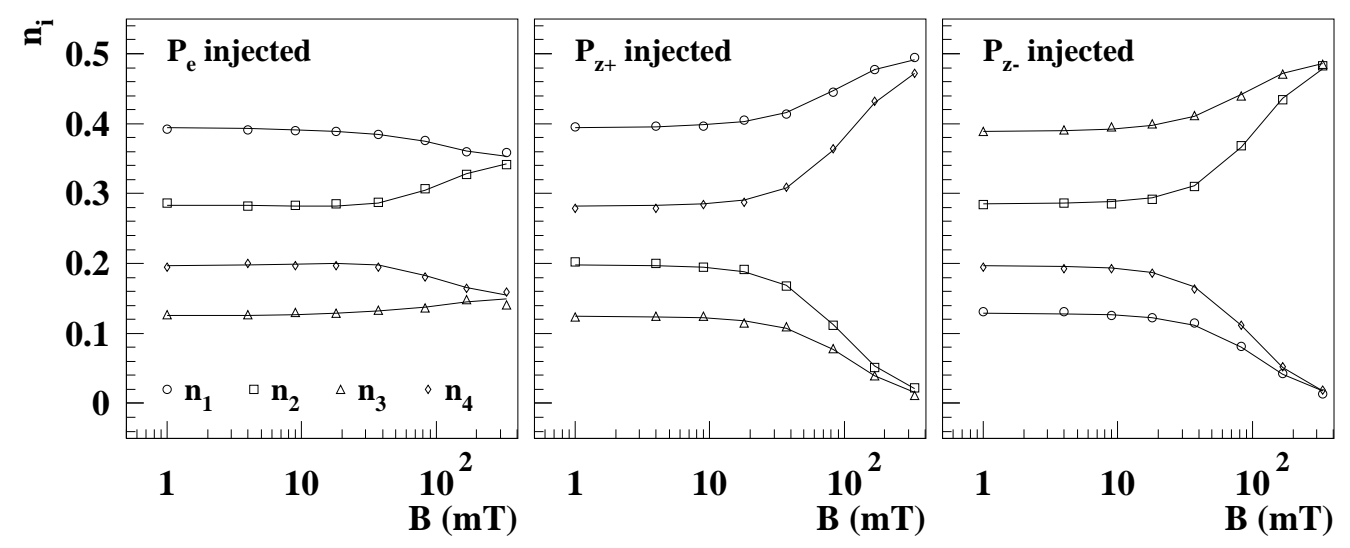

Fig. 11. Measured fractional hyperfine populations vs. magnetic field for hydrogen for the various injection modes. The measured values are given by symbols, while the lines show a fit using the solutions of eq. (28)

ulations $n_{i}$ of the hyperfine state $|i\rangle[16,30]^{12}$ :

$$
\frac{\mathrm{d} n_{i}}{\mathrm{~d} t}=\frac{1}{\tau_{d}}\left(n_{i}^{i n j}-n_{i}\right)+\frac{1}{\tau_{f}} \sum_{k} R_{j k} n_{k}+\frac{1}{\tau_{s e}} \sum_{j k} M_{j k}^{i} n_{j} n_{k} .
$$

The first two terms of eq. (28) characterize the incoming and outgoing fluxes, the third term represents the wall relaxation and the last term the spin exchange relaxation. The effect of recombination is not included, and it is assumed that the total flux from the ABS is constant with time. In eq. (28), three time constants appear: $\tau_{d}$ (the diffusion time) is the mean occupation time of atoms within the storage cell, $\tau_{f}$ is the mean time of flight between two wall collisions and $\tau_{s e}$ is the mean time of flight between two spin exchange collisions. For times that are large compared to $\tau_{d}$, the relative hyperfine populations converge toward a dynamic equilibrium (steady state). As an example of the application of eq. (28), the solution of the system of linear equations in the steady state (with $\frac{\mathrm{d} n_{i}}{\mathrm{~d} t}=0$ ) was solved directly in the case of hydrogen or with the help of a numerical iteration algorithm in the case of deuterium [16]. Fig. 11 represents the measured hyperfine fractional populations as a function of the magnetic field for hydrogen and a fit to the data using the solutions of eq. (28). The fitted parameters are the three time constants and optionally the injected atomic state fractional populations. The $R$ and $M$ transition probability matrices are calculated on the basis of theoretical models [16]. These data show that a holding field greater than $300 \mathrm{mT}$ is needed to have sizable polarization for the case of two injected states.

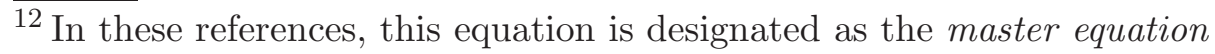




\subsubsection{Bunch field induced depolarization}

The beam of the HERA electron storage ring is bunched with time period $\tau_{\text {bunch }}=96.1 \mathrm{~ns}$ between two adjacent bunches. Typically, most of the $220 \mathrm{RF}$ buckets are filled, so that the bunch frequency is given by:

$$
\nu_{\text {bunch }}=\frac{1}{\tau_{\text {bunch }}}=10.42 \mathrm{MHz} .
$$

A large number of harmonics contribute to the induced magnetic high frequency field close to the HERA beam, because the bunches are very short $(\sigma=30 \mathrm{ps})$. The transition frequency between two hyperfine states $|i\rangle$ and $|j\rangle$ is given by:

$$
\nu_{i j}=\frac{E_{i}(B)-E_{j}(B)}{h} .
$$

If $\nu_{i j}$ matches one of these harmonics at a certain holding field $B$, resonant depolarization occurs. The location of the beam-induced resonances is shown in Fig. 12. Bunch field induced depolarization at the HERMES target has been studied in the cases of hydrogen $[13,33]$ and deuterium [16] for the longitudinal orientation of the target holding field (before 2001). During this time the observed $\pi(\Delta m= \pm 1)$ transitions were sufficiently well separated so that at the working field $(335 \mathrm{mT})$ no beam induced depolarization could be found. In the case of the transverse field that has been in use since October 2001, both $\sigma(\Delta m=0)$ and $\pi(\Delta m= \pm 1)$ transitions are allowed because the oscillating field surrounding the HERA beam has components both parallel and perpendicular to the static holding field. The spacing between two adjacent resonances is narrower than in the longitudinal case (only $0.37 \mathrm{mT}$ difference in the magnetic holding field at $B=0.3 \mathrm{~T}$ ), so a better homogeneity in the holding field is needed (see Fig. 12).

Thus a transverse field value for which beam resonant depolarization can be completely suppressed does not exist. A pair of additional correction coils mounted closely around the storage cell to improve the field homogeneity was installed for the 2003 running to further reduce resonant depolarization. The results will be presented in a forthcoming paper.

\section{Target polarization}

The average polarization $P_{T}$ of the nucleons in the target is a fundamental parameter of the HERMES experiment. The problem is to relate the measured atomic polarizations in the sample beam with the density-weighted average polarization of the nuclei in the cell. The determination of the target polarization involves several terms that mainly account for two effects: 


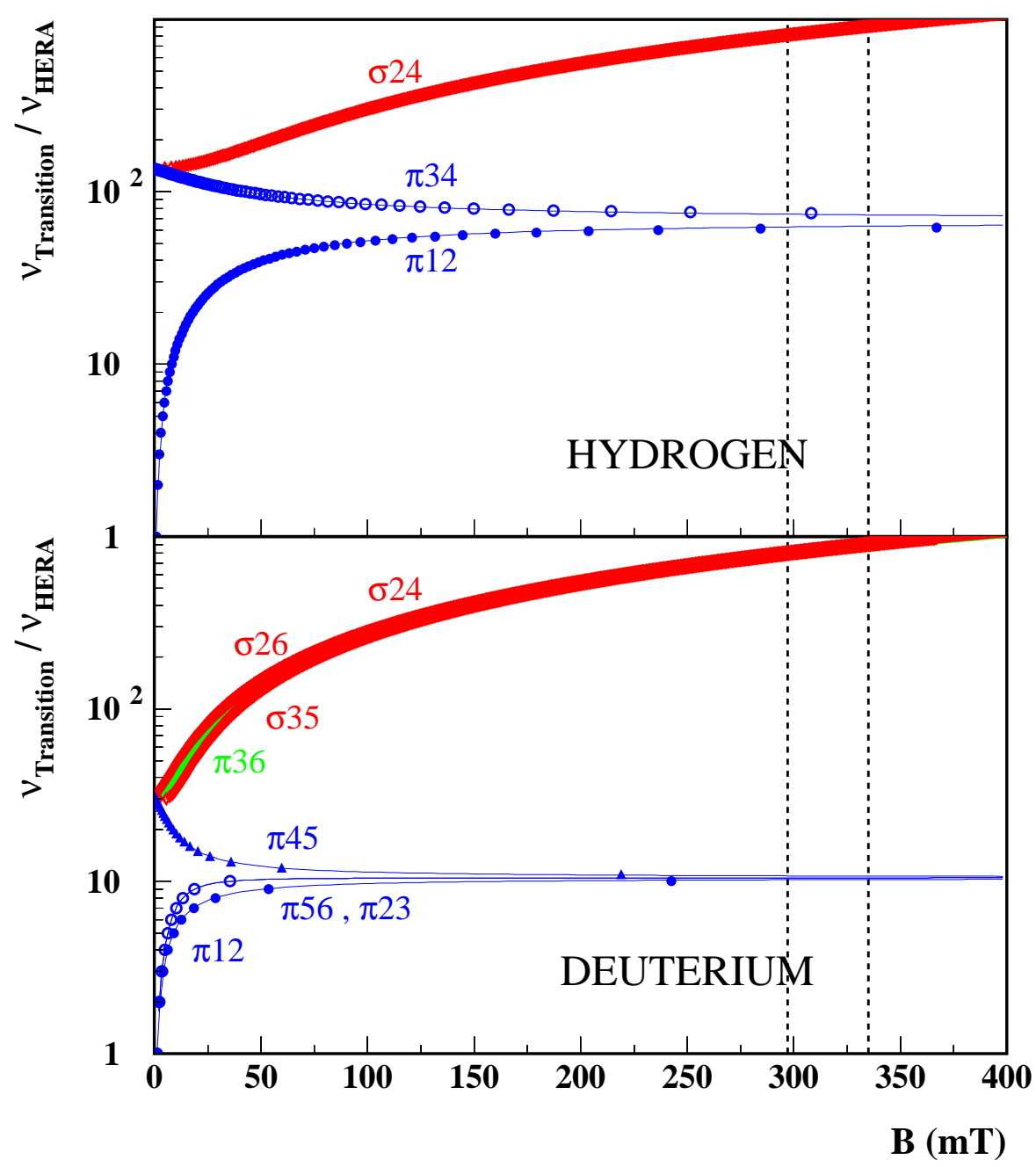

Fig. 12. Possible beam-induced nuclear depolarizing resonances in the HERMES target. The frequency difference between pairs of hyperfine states whose transitions lead to nuclear depolarization are plotted as a function of the holding field. The frequency values are normalized to $\nu_{H E R A}=10.42 \mathrm{MHz}$. The symbols representing the resonance conditions are clearly distinguishable for the $\pi$ transitions, while they overlap with each other in the case of $\sigma$ and $\pi|3\rangle \leftrightarrow|6\rangle$ transitions, which are separated by a difference in the intensity of the holding field $\Delta B_{T}$ of only $0.37 \mathrm{mT}$. The dashed lines represent the working fields of the transversely (left) and longitudinally (right) polarized targets.

- Presence of molecules in the cell, both unpolarized from the residual gas of the scattering chamber and/or directly injected from the ABS, and polarized molelcules coming from recombination of polarized atoms on the cell surface.

- Relaxation and recombination effects in the storage cell leading to an inhomogeneous distribution of the atomic fraction and of the atomic polarization. 
The average target polarization $P_{T}$ as seen by the electron beam is given by the following equation:

$$
P_{T}=\alpha_{0} \alpha_{r} P_{a}+\alpha_{0}\left(1-\alpha_{r}\right) \beta P_{a}
$$

where $\alpha_{0}$ represents the initial fraction of nucleons in atoms in the gas injected by the ABS (or atomic fraction in the absence of recombination), $\alpha_{r}$ denotes the fraction of nucleons in atoms that have survived recombination, and $\beta=$ $P_{m} / P_{a}$ represents the ratio of the nuclear polarization of the molecules $P_{m}$ produced by recombination relative to the nuclear polarization of the atoms $P_{a}$. The knowledge of $\beta$ for the experimental conditions at the HERMES experiment will be discussed in Sec. 5.2.

The values for $\alpha_{0}, \alpha_{r}$ and $P_{a}$ are calculated using the measurements of the TGA and BRP combined with various calibrations. As both detectors measure the properties of a sample of the target gas from the center of the target cell, it is necessary to relate the results to a corresponding value averaged along the storage cell. This is achieved by evaluating the sampling corrections [15]:

$$
\begin{aligned}
c_{\alpha} & \equiv \frac{\alpha_{r}}{\alpha_{r}^{T G A}}, \\
c_{P} & \equiv \frac{P_{a}}{P_{a}^{B R P}} .
\end{aligned}
$$

The sampling corrections and their systematic uncertainties are functions of the measured values of $\alpha_{r}^{T G A}$ and $P_{a}^{B R P}$, respectively. The functions depend on the geometry of the storage cell, its surface properties and the detectors' acceptances. They are calculated with the help of Monte Carlo simulations of the stochastic motion of particles in the storage cell, where the histories of these particles are stored and analyzed. Another possibility is to calculate sampling corrections analytically by describing the diffusion process of the target gas inside the storage cell with the use of a one-dimensional diffusion equation. The issue was addressed in Ref. [15] and will be discussed here in Sec. 5.4.

The various quantities entering the calculation of the average polarization are summarized in Tab. 6.

\subsection{Determination of the quantities $\alpha_{r}$ and $\alpha_{0}$}

In order to calculate the target polarization following eq. (31), it is necessary to separate the atomic fraction $\alpha_{0}$ that would be seen by the HERA electron beam if no cell wall recombination were to occur and the fraction $\alpha_{r}$ of the initially injected atoms that survive wall recombination, as seen by the HERA 


\begin{tabular}{|c|c|}
\hline Quantity & Meaning \\
\hline \hline$P_{T}$ & density-averaged nuclear polarization in the cell \\
\hline$\alpha_{0}$ & atomic fraction in the absence of recombination within the cell \\
\hline$\alpha_{r}=c_{\alpha} \alpha_{r}^{T G A}$ & fraction of atoms surviving recombination in the cell \\
\hline$c_{\alpha}$ & sampling correction for the atomic fraction \\
\hline$\alpha_{r}^{T G A}$ & measured atomic fraction in the sample beam \\
\hline$P_{a}=c_{P} P_{a}^{B R P}$ & nuclear polarization of atoms in the cell \\
\hline$c_{P}$ & sampling correction for the atomic polarizations \\
\hline$P_{a}^{B R P}$ & measured nuclear polarization of atoms in the sample beam \\
\hline$\beta=P_{m} / P_{a}$ & relative nuclear polarization of recombined atoms \\
\hline
\end{tabular}

Table 6

Meaning of the quantities contained in eq. (31).

electron beam. The measured quantity $\alpha^{T G A}$ can also be factored in two terms and can be written as (see eq. (9)):

$$
\alpha^{T G A}=\frac{\phi_{a}}{\phi_{a}+\phi_{m}}=\alpha_{0}^{T G A} \alpha_{r}^{T G A}
$$

Since dissociative ionization can be neglected [10], the only source of atomic flux $\phi_{a}$ into the gas analyzer is that originating from the atomic beam source. The molecular flux $\phi_{m}$ may come from three different sources:

- Undissociated molecules ballistically injected by the ABS. Since the efficiency of the dissociator is around $80 \%$, a significant number of molecules pass through the nozzle towards the polarizing stage, affected by neither the sextupole magnets nor the transition units. Although the majority of these molecules hit the vacuum chamber wall and are pumped away, a small number enter the storage cell via the injection tube. These unpolarized molecules have a triangular density distribution along the cell axis, with the maximum density at the center of the cell.

- Rest gas in the target chamber. These molecules originate mainly from the recombination of atoms that have left the storage cell and have thermalized on the vacuum chamber walls. This molecular component is clearly unpolarized and results in a uniform density component inside the storage cell.

- Atoms that recombine into molecules while in the cell. As explained in Sec. 2.3, the cell material and temperature are optimized in order to limit the depolarization and recombination effects in the target atomic gas. Nevertheless, atoms can stick on the cell surface and recombine, and the resulting molecules may carry a residual nuclear polarization. 
The normalized total molecular flow rate $\phi_{m}$ of atoms into the TGA ionizer can be decomposed in the following way:

$$
\phi_{m}=\phi_{b a l}+\phi_{r g}+\phi_{r}
$$

where $\phi_{b a l}, \phi_{r g}$ and $\phi_{r}$ represent the ballistic, the rest gas and the recombination components, respectively. To separate between polarized and unpolarized molecules, we rewrite the two factors in eq. (34) using the three contributions appearing in eq. (35):

$$
\begin{aligned}
\alpha_{r}^{T G A} & \equiv \frac{\phi_{a}}{\phi_{a}+\phi_{r}}, \\
\alpha_{0}^{T G A} & \equiv \frac{\phi_{a}+\phi_{r}}{\phi_{a}+\phi_{r}+\phi_{b a l}+\phi_{r g}} .
\end{aligned}
$$

The independent measurement of each of the three components of the total molecular flow rate is accomplished by means of routine target calibrations, which are usually performed between two HERA electron fills when the HERMES spectrometer is not taking data. The rest gas contribution $\phi_{r g}$ to the molecular flux is linearly proportional to the pressure in the target chamber. The constant of proportionality is determined by injecting into the target chamber increasing quantities of hydrogen (deuterium) molecules with a controlled flow system and by measuring the corresponding TGA count rates. The ballistic flux contribution $\phi_{b a l}$ can be measured by varying the atomic flux of the injected beam while keeping the molecular flux constant. The atomic flux is changed by using the hyperfine transitions to select either 1 or 2 (or 3 in the case of deuterium) hyperfine states. Since the recombination probability inside the cell was found to be independent of the injected beam intensity [12], the quantity $\phi_{b a l}$ can be extracted. Eq. (35) is then solved for the remaining molecular flux $\phi_{r}$. After the molecular contributions $\phi_{r g}, \phi_{b a l}$ and $\phi_{r}$ are extracted, one can determine $\alpha_{r}^{T G A}$ using eq. (36) and thus $\alpha_{r}$ using eq. (32) with the sampling correction $c_{\alpha}$ determined as described in Sec. 5.4. A detailed description of how these calibrations are performed was given in Ref. [10].

The factor $\alpha_{0}$ is defined in terms of the nucleon thicknesses $t_{a, r, b a l, r g}$ in the cell seen by the HERA beam:

$$
\alpha_{0} \equiv \frac{t_{a}+t_{r}}{t_{a}+t_{r}+t_{b a l}+t_{r g}}
$$

It can be determined without reference to additional sampling corrections. All the $\phi_{i}$ terms in eq. (37) represent nucleon fluxes. Let us define a proportionality constant $c$ representing the TGA response to atoms through the relationship $\phi_{a}=c t_{a}$. The fluxes $\phi_{r}, \phi_{b a l}$ and $\phi_{r g}$ are suppressed by a factor of $\sqrt{2}$ due to the smaller molecular velocity, leading to $\phi_{r}=c t_{r} / \sqrt{2}$ and $\phi_{b a l}=c t_{b a l} / \sqrt{2}$. 
Furthermore, in the limiting case of small cell wall recombination, the densities of atoms and molecules producing $t_{a}, t_{b a l}$ and $t_{r}$ are triangular. In contrast, the rest gas producing $t_{r g}$ has a uniform density along the cell, resulting in $\phi_{r g}=c t_{r g} /(2 \sqrt{2})$. If the difference between the conductances of the atoms and molecules is neglected so that they have the same distributions along the cell, the above considerations can be combined to give

$$
\alpha_{0}=\frac{\phi_{a}+\sqrt{2} \phi_{r}}{\phi_{a}+\sqrt{2}\left(\phi_{r}+\phi_{b a l}+2 \phi_{r g}\right)} .
$$

However, this difference in conductances can be taken into account [16,24], leading to different distributions along the cell and a more complicated expression:

$$
\alpha_{0}=\frac{d\left(\phi_{a}+\phi_{r}\right)}{d\left(\phi_{a}+\phi_{r}\right)+\sqrt{2}\left(\phi_{b a l}+2 \phi_{r g}\right)},
$$

where $d=\sqrt{2} /\left(1+(\sqrt{2}-1) \alpha_{r}\right)$.

\subsection{Relative polarization $\beta$ of nuclei in recombined atoms}

As mentioned in the previous sections, some of the polarized hydrogen or deuterium atoms may recombine in the storage cell. A direct measurement of the remnant polarization contained in the molecules is not possible at HERMES, as the Breit-Rabi polarimeter can measure the atomic polarizations only. Atomic recombination is a surface-mediated temperature-dependent process $[12,16]$. Hence measurements of the nuclear polarization of recombined atoms using storage cells of various materials and/or with various coatings $[37,38]$ are not directly applicable to the HERMES case. Without any information about the polarization $\beta$ of nucleons in molecules (or molecular polarization in short) one has to allow in eq. (31) the conservative range $0 \leq \beta \leq 1$. A set of data taken in 1997 at a higher cell temperature $(260 \mathrm{~K}$ instead of the nominal $100 \mathrm{~K}$ ) was used to measure $\beta$ for hydrogen at that temperature, resulting in $\beta^{260 K}=0.68 \pm 0.09_{\text {stat }} \pm 0.06_{\text {syst }}[14]$. This result can be used as an upper limit for $\beta$ at $100 \mathrm{~K}\left(\beta_{\text {high }}^{100 K}\right)$ under the following assumptions. The main mechanism responsible for recombination at $100 \mathrm{~K}$ and $260 \mathrm{~K}$ is the same (i.e. the E-R mechanism) [12]. At $100 \mathrm{~K}$, the recombination probability is smaller than at $260 \mathrm{~K}$, because the atoms impinging from the volume have less kinetic energy to overcome the activation barrier. The residence time of chemically adsorbed atoms on the surface at $100 \mathrm{~K}$ is greater than at $260 \mathrm{~K}$, and their possible polarization cannot exceed the value measured at $260 \mathrm{~K}$. This results in $\beta_{\text {high }}^{100 K}=0.83$.

The lower limit for $\beta^{100 K}\left(\beta_{\text {low }}^{100 K}\right)$ can be derived from a simple argument. By assuming that the nucleon spins are not affected by the recombination process, 
the nuclear polarization of the molecule at the time of its formation $\left(P_{m}^{0}\right)$ can be evaluated by taking the average value of the polarization of an atom coming from the volume $\left(P_{a}\right)$ and one resident on the surface $\left(P_{s}\right)$. The theoretical

lower limit for $\beta^{100 K}$ can then be inferred by supposing that atoms sticking on the cell surface are totally depolarized $\left(P_{s}^{100 K, l o w}=0\right)$ and applying the equation:

$$
\begin{aligned}
\beta_{\text {low }}^{100 K} & =\frac{P_{m}^{100 K, \text { low }}}{P_{a}^{100 K}} \\
& =\frac{P_{a}^{100 K}+P_{s}^{100 K, \text { low }}}{2} \frac{1}{P_{a}^{100 K}} .
\end{aligned}
$$

The result is $\beta_{\text {low }}^{100 K}=0.5$. A factor of 0.9 takes into account the depolarization of molecules while colliding with the walls $[14,38]$, ending in $\beta_{\text {low }}^{100 K}=0.45$. Hence, the range of $\beta^{100 K}$ can be limited to

$$
0.45 \leq \beta^{100 K} \leq 0.83
$$

The corresponding value and uncertainty of $\beta^{100 K}$ are therefore given by:

$$
\beta^{100 K}=0.64 \pm 0.19 .
$$

\subsection{Injected atomic polarizations}

An important parameter for the evaluation of the target polarization is the injected atomic polarization $P_{a}^{i n j}$ (i.e. the nuclear polarization of atoms in the absence of depolarization), as it enables the separation of the various spinrelaxation effects. The injected polarization for each ABS operating mode (Tab. 2) can be calculated if the transmission probabilities of the sextupole system and the efficiencies of the adiabatic transition units are known. The sextupole transmission probabilities for hydrogen and deuterium have been calculated using a Monte Carlo simulation and the results are given in Ref. [8]. The injected polarization is fairly insensitive to these values. The efficiencies of the transitions of the ABS can be extracted by measuring the magnetic field dependence of the spin relaxation processes and by fitting the data with a theoretical model described in Sec. 4.2 .3 and given by eq. (28). For normal operation, the important ABS injection modes are those having a large positive or negative nuclear polarization, $P_{z+}$ and $P_{z-}$ respectively, and, in the case of deuterium, the two additional positive and negative tensor polarization $\left(P_{z z+}\right.$ and $P_{z z-}$ ) modes (see Tab. 2).

For hydrogen, only two transitions after the sextupole system are used: the SFT 2-4 for $P_{z+}$ and WFT 1-3 for $P_{z-}$. Therefore, the injected polarizations are 
directly given by the transition efficiencies whose values have been extracted in Ref. [16] with the technique mentioned above, and cross-checked using a more direct method based on the symmetry of the spin relaxation processes for the two injection modes. In the case of deuterium, several transition units are used to provide the four polarizations, some of which are located between the two sextupole subsystems. Hence, the injected polarizations have to be calculated taking into account the transition efficiencies as well as the transmission probabilities for atoms whose hyperfine state gets changed between the two subsystems.

The calculated polarization for the hydrogen and deuterium beams injected into the target are summarized in Tab. 7. The degree of nuclear polarization injected by the ABS when running with deuterium is substantially lower than for hydrogen. The reason is that, due to the higher number of hyperfine states, the deuterium nuclear polarization can be achieved only by using the transition units located between the two magnetic subsystems, resulting in an incomplete separation of states.

\subsection{Sampling Corrections}

The sampling correction functions $c_{\alpha}\left(\alpha_{r}^{T G A}\right)$ and $c_{P}\left(P_{a}^{B R P}\right)$ depend strongly on the distribution of recombination and wall relaxation probabilities per wall collision along the storage cell. For a freshly produced storage cell that has not yet been exposed to the HERA beam, one can assume that the recombination and wall relaxation probabilities are constant over the entire surface of the storage cell, or at least that microscopic inhomogeneities due to various types of surface sites are equally distributed. Possible mechanisms by which the HERA beam could affect the surface properties of the storage cell include:

\section{Table 7}

\begin{tabular}{|c|c|c|}
\hline Mode & Hydrogen & Deuterium \\
\hline \hline$P_{z+}^{i n j}$ & $97.26 \pm 0.05$ & $87.78 \pm 0.07$ \\
\hline$P_{z-}^{i n j}$ & $-97.38 \pm 0.07$ & $-89.50 \pm 0.10$ \\
\hline$P_{z z+}^{i n j}$ & - & $96.22 \pm 0.38$ \\
\hline$P_{z z-}^{i n j}$ & - & $-177.30 \pm 0.61$ \\
\hline
\end{tabular}

Estimated polarizations for the hydrogen and deuterium atomic beams injected by the ABS into the target. All values are multiplied by 100. The quoted uncertainties take only the dominant uncertainties of the ABS efficiencies into account. The values refer to the polarization of injected gas with the $0.3 \mathrm{~T}$ holding field. Note that, due to the standard way of defining it, the maximum achievable negative tensor polarization is -200 . 
- desorption of the surface coating by synchrotron radiation;

- bombardment of the storage cell walls with charged particles leading to radiation damage;

- chemical reactions of ionized hydrogen or deuterium radicals with the cell surface;

- redistribution of the ice layer on the storage cell by RF heating due to the wake fields;

- contamination of the storage cell surface by sputtered material from the collimator upstream of the target.

Under normal operating conditions no influence of the HERA beam on the target performance has been observed. Nevertheless certain distinct incidents affecting the cell have been observed, when the beam tune was not optimal or the HERA beam was accidentally lost near the HERMES target region. Since the effect of the HERA beam on the surface properties is poorly known, every realistic distribution of recombination and wall relaxation probabilities has to be considered when evaluating the sampling corrections. It is therefore convenient to distinguish certain scenarios of special interest.

- Homogeneous cell (HC): the natural assumption for a freshly produced cell not yet exposed to the HERA beam. The calculated sampling corrections set an upper limit for $\alpha_{r}$ and $P_{a}$ for any given values of $\alpha_{r}^{T G A}$ and $P_{a}^{B R P}$, respectively.

- Homogeneous beam tube (HBT): the main cell tube is homogeneously affected by the beam, while the side tubes are unaffected.

- Inhomogeneous beam tube (IBT): The main cell tube is inhomogeneously affected by the beam, while the side tubes are unchanged. This scenario does not lead to a single sampling correction, but it can be used to calculate a lower limit for $\alpha_{r}$ or $P_{a}$ for any given value of $\alpha_{r}^{T G A}$ or $P_{a}^{B R P}$, respectively.

A model for calculating the sampling corrections was developed in Ref. [16]. It is based on a parameter $\gamma_{r}\left(\gamma_{d}\right)$, the recombination (spin-flip) probability during a wall collision. If these probabilities were constant and equal for the beam and sample tubes, the sampling corrections would depend only on their geometry. This is actually the case for a freshly installed cell, whose Drifilm coating is known to be intact and uniform. In all scenarios it is assumed that the relation $\gamma_{r}^{S T} \leq \gamma_{r}^{B T}$ holds at any time for the recombination probabilities in the sample tube, $\gamma_{r}^{S T}$, and beam tube, $\gamma_{r}^{B T}$. The validity of this hypothesis is proven by the data $[16,24]$. The numerical calculations of the sampling corrections have been performed using a molecular flow simulation, complemented by analytic calculations based on the one-dimensional diffusion equation [16].

The sampling correction $c_{P}$ must be subdivided into two terms, one for the wall collisions and a second term for spin exchange relaxation, as the two 
processes must be treated in different ways. This can be done by defining the depolarization factors $D_{w d}$ (wall collisions) and $D_{s e}$ (spin exchange relaxation) so that for small spin relaxation, one has:

$$
c_{P} \equiv \frac{P_{a}}{P_{a}^{B R P}} \simeq \frac{D_{w d} D_{s e} P_{a}^{i n j}}{D_{w d}^{B R P} D_{s e}^{B R P} P_{a}^{i n j}} .
$$

As the spin exchange relaxation occurs in the gas phase, and for the typical values of $\alpha_{r}>0.9$ the atomic density distribution is little affected by any change of the cell surface properties, the relation between $D_{s e}$ and $D_{s e}^{B R P}$ is similar for all of the listed scenarios. Moreover, since calculations have shown that $D_{s e} \simeq D_{s e}^{B R P}[16]$, one can conclude that the sampling correction $c_{P}$ is determined by the wall depolarization only:

$$
c_{P}=\frac{P_{a}}{P_{a}^{B R P}} \simeq \frac{D_{w d}}{D_{w d}^{B R P}} .
$$

As an example, in Fig. 13 the effect of the calculated sampling corrections for the large-aperture cell used in the 1996-97 running with hydrogen is shown. During normal operation, the values of $\alpha_{r}^{T G A}$ and $D_{w d}^{B R P}$ are found to be almost always well above 0.9 , so that local linear approximations may be fitted to the calculated sampling corrections:

$$
\begin{aligned}
\alpha_{r} & =\bar{a}+(1-\bar{a}) \alpha_{r}^{T G A} \\
& =\alpha_{r}^{T G A}+\bar{a}\left(1-\alpha_{r}^{T G A}\right), \\
D_{w d} & =\bar{b}+(1-\bar{b}) D_{w d}^{B R P} \\
& =D_{w d}^{B R P}+\bar{b}\left(1-D_{w d}^{B R P}\right) .
\end{aligned}
$$

The values of the parameters $\bar{a}$ for recombination and $\bar{b}$ for depolarization are fitted to the average between the two extreme scenarios shown in Fig. 13, and their systematic uncertainties $\Delta \bar{a}$ and $\Delta \bar{b}$ are taken to be the differences found when fitting the extremes.

\subsection{Evaluation of the systematic uncertainty in $P_{T}$}

After having determined all the terms of eq. (31), $P_{T}$ can be expressed as a function of the measured quantities. It is convenient to write the target polarization in the following way:

$$
P_{T}=\alpha_{0}\left(\alpha_{r}+\left(1-\alpha_{r}\right) \beta\right) P_{a}=\alpha_{e f f} \cdot P_{a}
$$

where the term $\alpha_{\text {eff }}$ is called effective atomic fraction. 

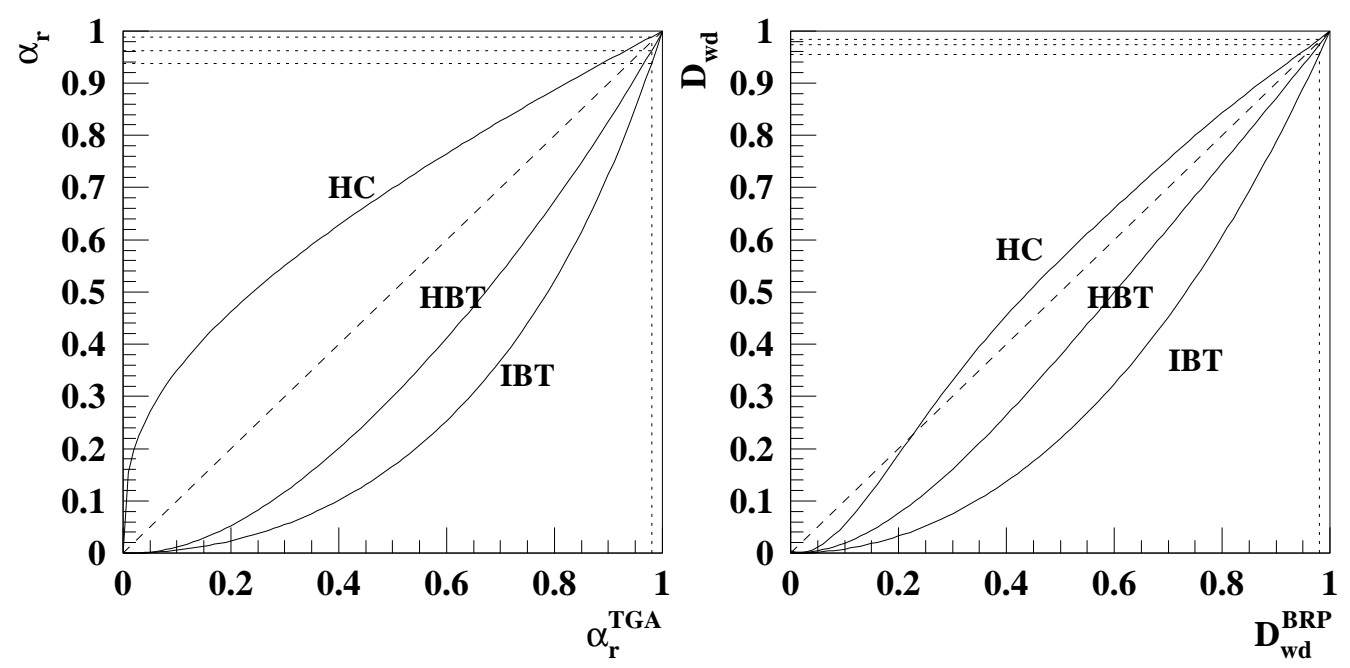

Fig. 13. Simulated correlation between the values measured by the TGA or BRP detectors and their corresponding density-weighted average values in the storage cell. The three solid curves represent the three scenarios for the state of the cell surface: homogeneous cell (HC), homogeneous beam tube (HBT), and inhomogeneous beam tube (IBT). The left figure shows the range for recombination and the right picture shows the range for wall depolarization. Acceptable uncertainties of $\alpha_{r}$ and $D_{W D}$ (differences among the three horizontal dotted lines) can be achieved only with $\alpha_{r}^{T G A}$ and $D_{W D}^{B R P}$ close to unity (vertical dotted lines). The corresponding uncertainty grows rapidly with increasing recombination or depolarization.

\subsubsection{Uncertainty of the effective atomic fraction $\alpha_{\text {eff }}$}

Combining eqs. (50), (47), (40), (36) and (35), $\alpha_{\text {eff }}$ can be expressed as

$$
\alpha_{e f f}=\frac{\phi_{a}+(\bar{a}(1-\beta)+\beta)\left(\phi_{m}-\phi_{b a l}-\phi_{r g}\right)}{\phi_{a}+\phi_{m}+(\sqrt{2}-1) \phi_{b a l}+(2 \sqrt{2}-1) \phi_{r g}} .
$$

The systematic uncertainty of $\alpha_{\text {eff }}$ is determined by the measured flow rates $\phi_{a}, \phi_{m}, \phi_{b a l}$ and $\phi_{r g}$ and their uncertainties as well as the values and uncertainties of $\beta, \bar{a}$ and the TGA calibration constant $\kappa$. With the matrix $T$ defined by

$$
T=\left(\frac{\partial \alpha_{e f f}}{\partial \phi_{i}}, \ldots, \frac{\partial \alpha_{e f f}}{\partial \kappa}\right),
$$

and the diagonal covariance matrix $C$ of all calibration constants, the systematic uncertainty in $\alpha_{e f f}$ is given by

$$
\Delta_{s y s} \alpha_{e f f}=\sqrt{T C T^{T}} .
$$

The matrix $T$ contains 10 independent derivatives, i.e. for $\kappa, \bar{a}, \beta$ and the 7 additional terms related to the ballistic flux and rest gas calibrations as described in Ref. [10]. 


\subsubsection{Uncertainty of the atomic polarizations $P_{a}$}

Combining eqs. (44), (45) and (49), the average nuclear polarization $P_{a}$ of atoms in the HERA beam can be written as

$$
P_{a}=P_{a}^{B R P}+\bar{b}\left(D_{s e} P_{a}^{i n j}-P_{a}^{B R P}\right)
$$

For the calculation of the systematic uncertainty $\Delta_{\text {sys }} P_{a}$, the important uncertainty sources to be included are $\Delta \bar{b}$ and $\Delta_{\text {sys }} P_{a}^{B R P 13}$. As a result, one finds:

$$
\Delta_{s y s} P_{a}=\left((1-\bar{b})^{2}\left(\Delta_{s y s} P_{a}^{B R P}\right)^{2}+\left(1-D_{w d}\right)^{2} D_{s e}^{2}\left(P_{a}^{i n j}\right)^{2} \Delta \bar{b}^{2}\right)^{1 / 2} .
$$

During the analysis one typically averages over a large dataset, so that the statistical uncertainty of the polarization measurement $\Delta_{s t a} P_{a}$ is negligible.

\section{Performance}

In the present section an overview is given about the target performance in the running period 1996-2003, by analyzing distinct periods for each target:

- 1997 for the longitudinal hydrogen target;

- 2000 for the longitudinal deuterium target;

- 2002 for the transverse hydrogen target.

\subsection{Longitudinal hydrogen running in 1997}

The upper plot in Fig. 14 shows the atomic fraction $\alpha^{T G A}$ measured by the target gas analyzer during the 1997 running period. The nuclear polarization of atoms measured by the BRP is plotted in the lower half of Fig. 14. Aside from startup problems, the target performance was relatively smooth. Unfortunately, a severe HERA beam loss close to the HERMES region occurred at a certain point (left line), resulting in a change of the cell surface properties. After this bad event, it was decided to collect a set of data at higher temperature $\left(T_{\text {cell }}=260 \mathrm{~K}\right.$ instead of the nominal value $\left.T_{\text {cell }}=100 \mathrm{~K}\right)$ in order to measure the $\beta$ parameter (see Ref. [14]). At the end of this period, the storage cell was exchanged (middle line). Also the second cell experienced a beam loss (right line) which produced a small hole in the cell wall. For these reasons, the analysis of the 1997 dataset was divided into four periods, during each of which the cell surface condition was assumed to be constant. For each

${ }^{13}$ The uncertainties on $\Delta P_{s e}$ and on $P_{a}^{i n j}$ are negligible. 

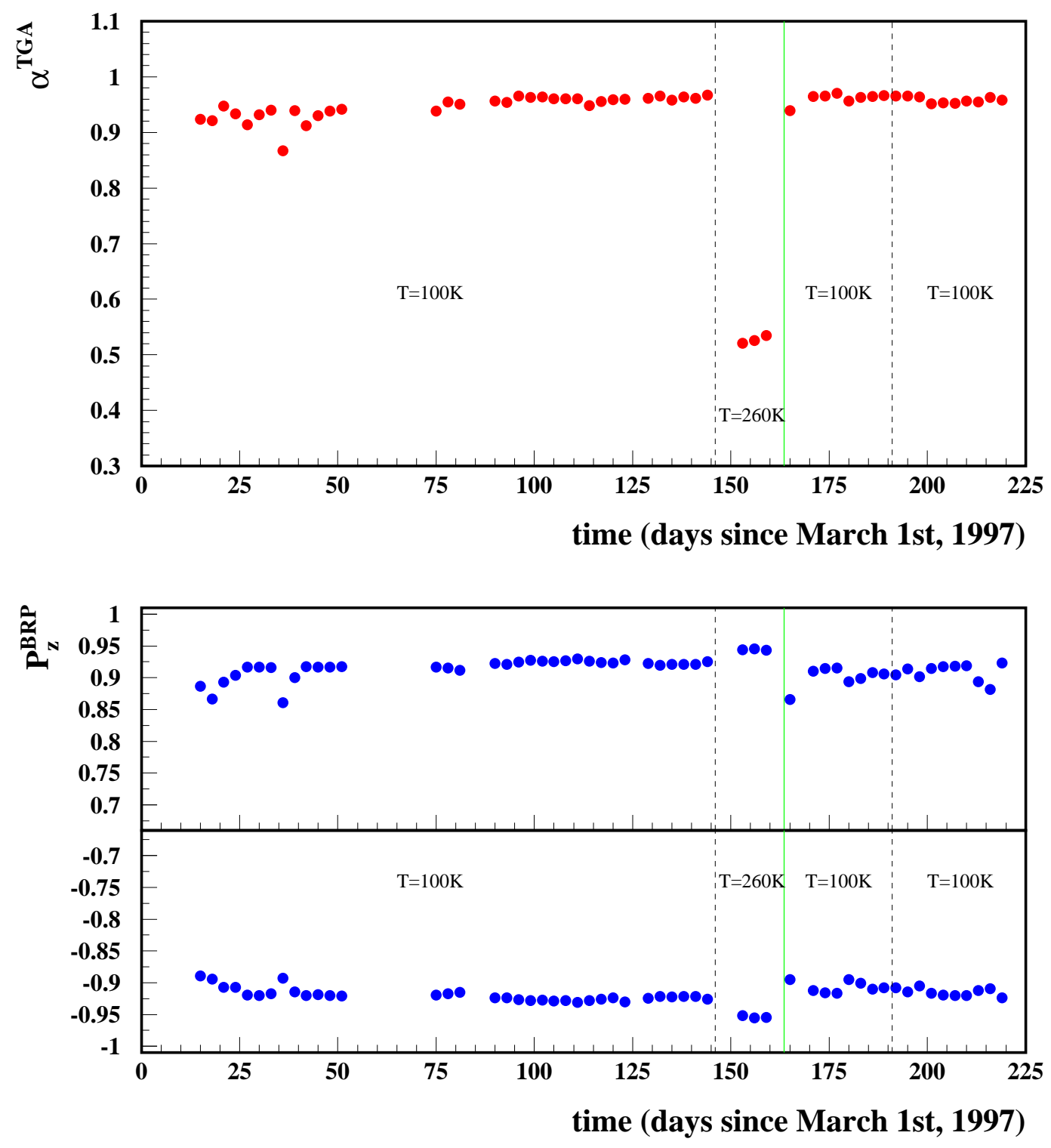

Fig. 14. The TGA (above) and BRP (below) measurements for the entire 1997 data taking period. The vertical dashed lines indicate HERA beam loss events which affected the cell surface properties, and the solid line indicates the replacement of the target cell.

period, specific studies have been performed to limit the uncertainties on $\alpha_{r}$ and $P_{a}[24]$.

For the entire running period with hydrogen, we quote a luminosity-weighted average value for the target polarization of $P_{z+}=-P_{z-}=0.852 \pm 0.033$ and a density of $7.6 \times 10^{13}$ nucl. $/ \mathrm{cm}^{2}$. The latter number was derived from a method exploiting the density dependence of spin exchange collisions [16]. 


\subsection{Longitudinal deuterium running in 2000}

Over the eight month period of deuterium running in 2000, the HERMES target operated under very stable conditions. The atomic beam source, equipped with a microwave dissociator, experienced only one major failure, which hardly affected data accumulation. Neither the scattering chamber nor the TGA/BRP vacuum system had to be opened, leading to constant amounts of residual gas in the storage cell as well as stable efficiencies of the detectors monitoring the target. There was no need to replace the storage cell, as its performance in terms of atomic recombination and nuclear depolarization was always excellent. The reason for such good behavior can be found both in a particularly fortunate Drifilm coating and in the limited damage caused by a very well tuned electron beam, i.e. no unwanted beam losses in the vicinity of the target.

The outstanding performance of the HERMES target in this period is well represented in Fig. 15 (note the change in scale compared to Fig. 14). The stable behavior of the cell surface in terms of atomic recombination is demonstrated in the upper plot of Fig. 15, where the atomic fraction measured by the target gas analyzer is plotted. The lower plot shows the nuclear polarization. In the period between January and June 2000, the target was operated with only positive and negative states of nuclear vector polarization, implying positive tensor polarization. From July on, a new injection mode combining vector and tensor polarization $P_{z+}, P_{z-}, P_{z z+}$ and $P_{z z-}$ was established in order to perform the first measurement of the tensor-polarized structure function $b_{1}^{d}$.

The average values for the four polarization states used in 2000 are the following:

$$
\begin{gathered}
P_{z+}=+0.851 \pm 0.029 \\
P_{z-}=-0.840 \pm 0.026 \\
P_{z z+}=+0.891 \pm 0.027 \\
P_{z z-}=-1.656 \pm 0.049
\end{gathered}
$$

Note that only the systematic uncertainty is quoted as the statistical uncertainty is negligible in comparison. The differences between the systematic uncertainties originate from the injection mode dependent systematic uncertainty of the atomic polarizations. The measured target density for this period, measured by means of the spin-exchange collision relaxation method, is $2.1 \times 10^{14}$ nucl. $/ \mathrm{cm}^{2}$. 

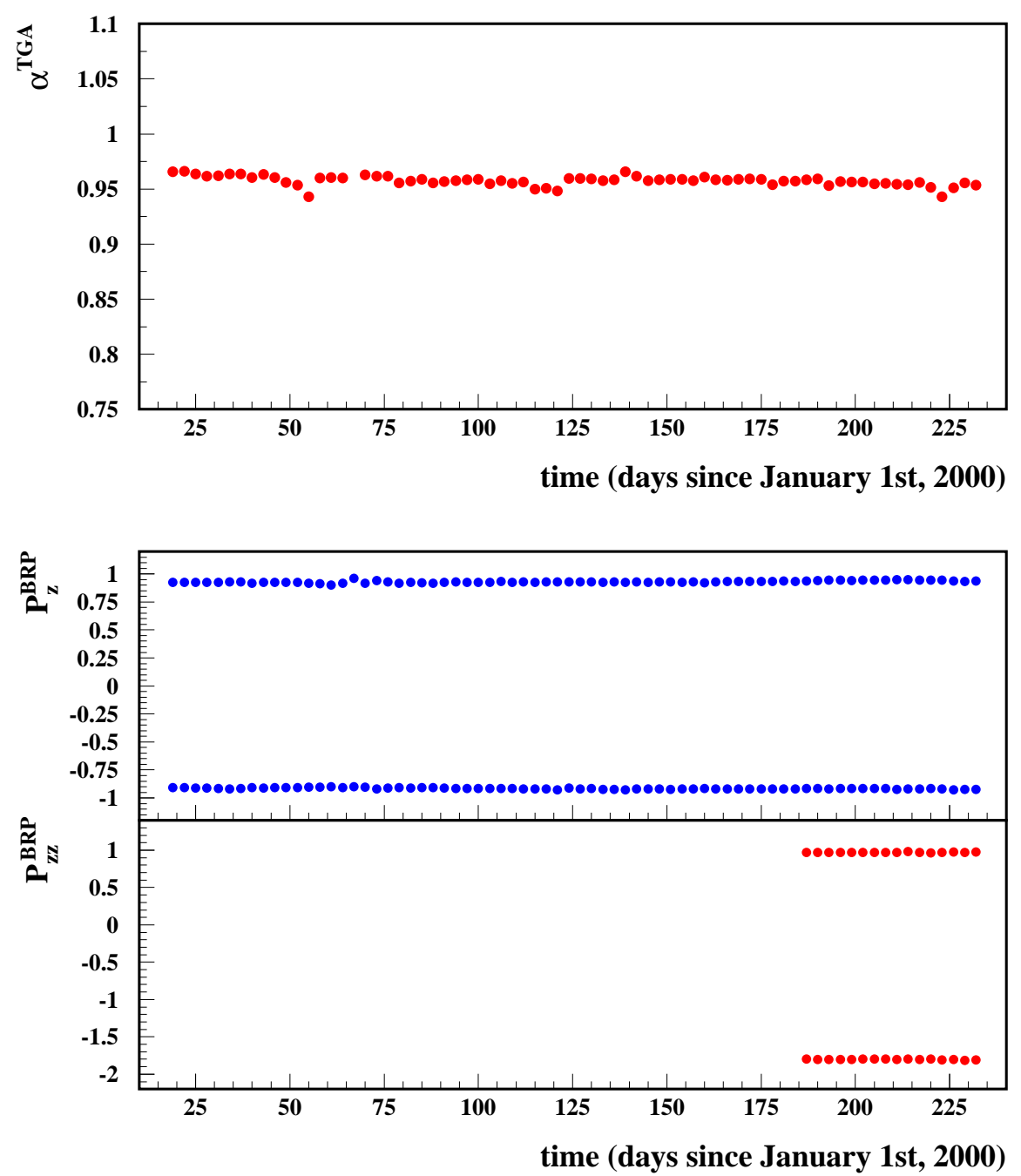

Fig. 15. Atomic fraction, $\alpha^{T G A}$ upper plot, and vector/tensor polarization, $P^{B R P}$ lower plots, measured by TGA and BRP respectively during the year 2000 running period. Each symbol represents data averaged over a 72-hour bin. The absolute bin averaged values are always above 0.9. The tensor polarization (lower plot) was employed from July on.

\subsubsection{Spin relaxation}

The spin-flip probability per wall collision $\gamma_{d}$ is proportional to $\tau_{s}$, the mean residence time: $\tau_{s}=\tau_{0} e^{\left(\frac{E_{b}}{k T}\right)}$ (see eq. $\left.(25)\right)$. Therefore $\gamma_{d}$ depends on the wall temperature $T$. In other words, if $P_{a}^{B R P}$ does not change with $T$, wall depolarization effects are vanishingly small within the experimental uncertainties. In Fig. 16, a scan of the cell temperature down to very low temperatures is shown. The result indicates that no wall depolarization occurs. As a consequence, for the deuterium target the sampling correction for nuclear depolarization equals unity $\left(c_{P}=1\right)$, so that:

$$
P_{a}=P_{a}^{B R P}
$$




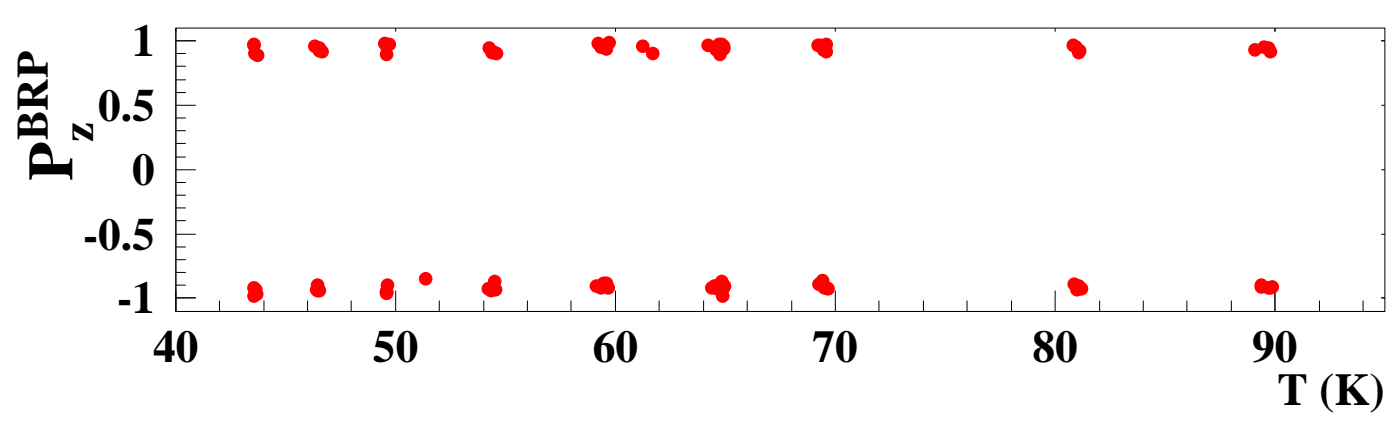

Fig. 16. Nuclear vector polarization $P_{z}^{B R P}$ as function of the cell temperature $\mathrm{T}$ measured in August 2000. No dependence on the temperature is observed. The operating temperature of the target during data collection was $60 \mathrm{~K}$.

Moreover, the calculated depolarization due to spin exchange collisions resulted in $\Delta P_{a}^{s e}=0.3 \%$. As in 2000 spin relaxation can be neglected completely, the polarization injected by the ABS equals the measured BRP value:

$$
P_{a}^{i n j} \simeq P_{a}^{B R P} .
$$

The polarizations measured by the BRP were found to be consistent with those calculated as described in Sec. 5.3, within the uncertainties.

\subsubsection{Recombination}

Although atomic recombination depends strongly on temperature, no variation of the measured atomic fraction $\alpha^{T G A}$ over a very wide temperature range around the working temperature $T_{\text {cell }}=60 \mathrm{~K}$ was ever observed during the whole year. Fig. 17 (upper plot) reports a cell temperature scan performed in August. (For comparison a temperature scan performed with hydrogen in 1997 is also shown in the lower plot of the same figure). Additionally the measurement of the fraction $\alpha_{r}^{T G A}$ of atoms surviving recombination yielded the following average value:

$$
\alpha_{r}^{T G A}=0.997 \pm 0.014
$$

confirming that within the measured uncertainty no evidence of recombination was found. Therefore, for the deuterium operating period in 2000, both the deviation from unity of the sampling correction $c_{\alpha}$, and the impact of the uncertainty in $\beta$ are negligible.

\subsection{Transverse hydrogen running in 2002}

During the 2002-2003 operating period, the HERMES transversely polarized hydrogen target worked in a very stable way. On the other hand, the poor 

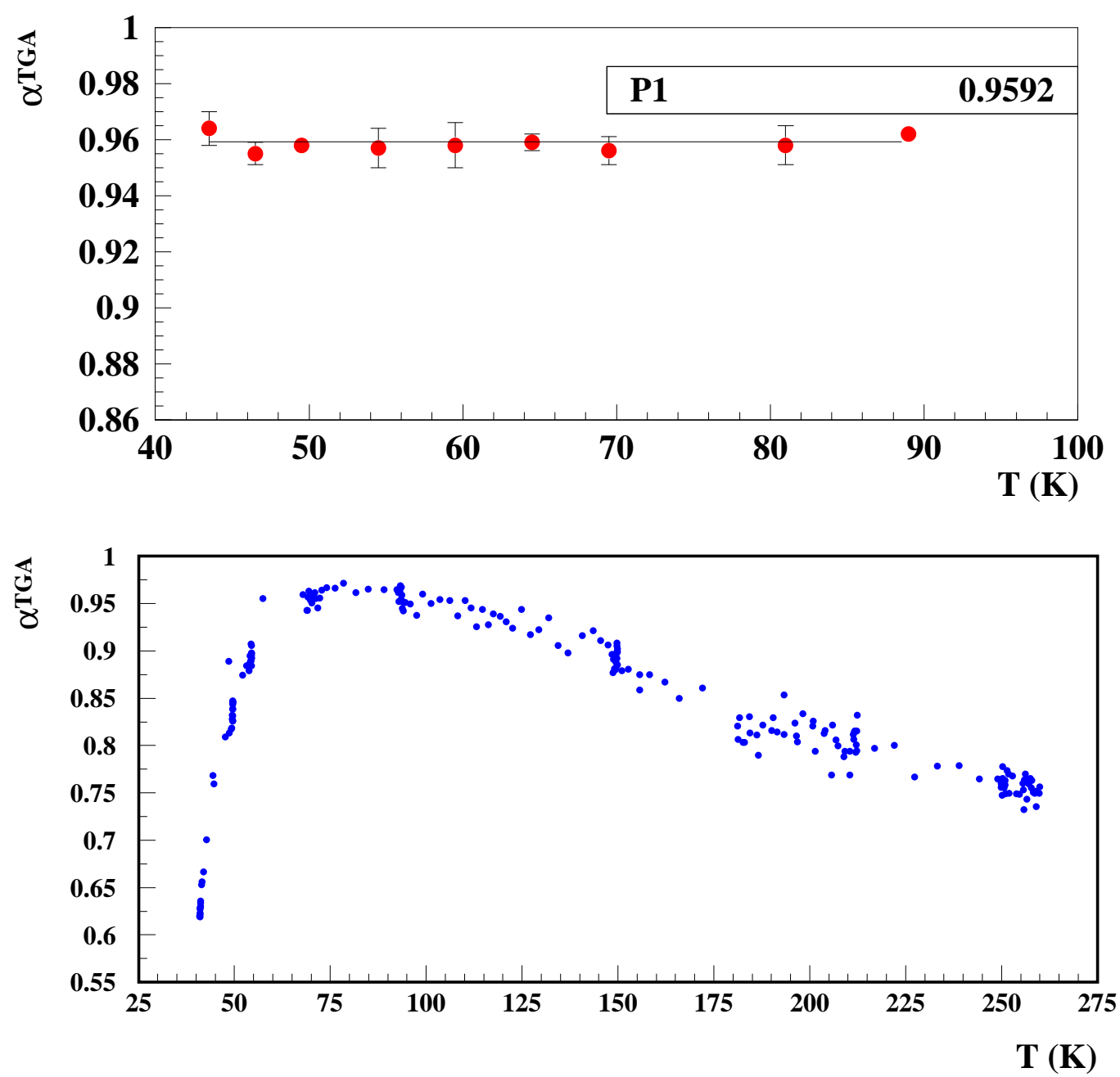

Fig. 17. Upper plot: measured atomic fraction as a function of the cell temperature for $T_{\text {cell }}<100 \mathrm{~K}$ for the deuterium running in 2000. No dependence on the temperature can be seen. The working temperature during normal operation was set to $T_{\text {cell }}=60 \mathrm{~K}$. For comparison a temperature scan with hydrogen taken in 1997 is shown in the lower plot.

performance of the HERA beam did not allow a detailed study of the bunch induced depolarization. Nevertheless, by scanning the target magnetic field a working field value was established where the loss of polarization due to the field non-uniformity was limited to $1.5 \%$ at the highest available beam currents $(25 \mathrm{~mA})$. The average target polarization $\left(P_{z+}-P_{z-}\right) / 2$ during the 2002 data taking period was $0.783 \pm 0.041$. This value is lower than that reported for the longitudinal hydrogen target (Section 6.1), mainly due to the lower applied target holding field and to the fact that the higher density allowed by the use of the intermediate size cell resulted in an increase of spin relaxation due to spin exchange collisions.

The stability of the transverse target operation during the 2002 running period 

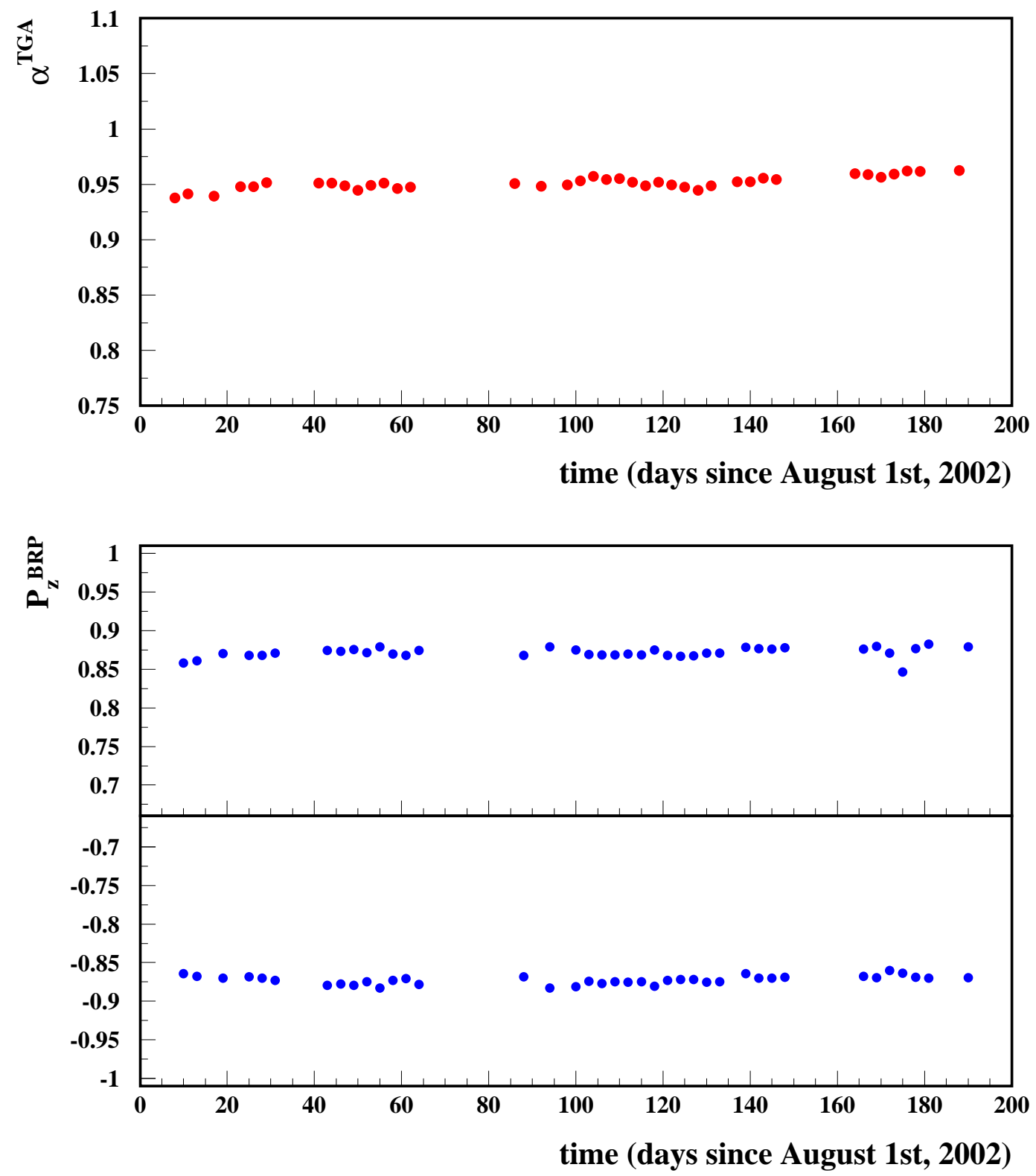

Fig. 18. The TGA (above) and BRP (below) measurements for the 2002/03 data taking period.

is illustrated in Fig. 18. The target density in this period measured via spinexchange collisions was $1.1 \times 10^{14}$ nucl. $/ \mathrm{cm}^{2}$.

\section{Discussion}

Tab. 8 summarizes averaged values for the target properties during the various running periods. A comparison between the longitudinally polarized hydrogen 


\begin{tabular}{|c|c|c|c|}
\hline & $H_{\|}(1997)$ & $H_{\perp}(2002)$ & $D_{\|}(2000)$ \\
\hline \hline$\alpha_{0}$ & $0.960 \pm 0.010$ & $0.918 \pm 0.032$ & $0.919 \pm 0.026$ \\
\hline$\alpha_{r}$ & $0.945 \pm 0.035$ & $0.979 \pm 0.023$ & $0.997 \pm 0.017$ \\
\hline$P_{z+}$ & $+0.908 \pm 0.016$ & $+0.859 \pm 0.032$ & $+0.927 \pm 0.017$ \\
\hline$P_{z-}$ & $-0.908 \pm 0.016$ & $-0.859 \pm 0.032$ & $-0.915 \pm 0.010$ \\
\hline$-\Delta P_{S E}$ & 0.035 & 0.055 & $\leq 0.001$ \\
\hline$-\Delta P_{W D}$ & 0.02 & 0.055 & $\leq 0.01$ \\
\hline$-\Delta P_{B I}$ & $\mathrm{absent}$ & 0.015 & $\mathrm{absent}$ \\
\hline$P_{+}$ & $+0.851 \pm 0.031$ & $+0.783 \pm 0.041$ & $+0.851 \pm 0.029$ \\
\hline$P_{-}$ & $-0.851 \pm 0.031$ & $-0.783 \pm 0.041$ & $-0.840 \pm 0.026$ \\
\hline$t\left(10^{14}\right.$ nucleons $\left./ \mathrm{cm}^{2}\right)$ & 0.7 & 1.1 & 2.1 \\
\hline FOM $\left(P^{2} t\right)\left(10^{14} / \mathrm{cm}^{2}\right)$ & 0.5 & 0.67 & 1.5 \\
\hline
\end{tabular}

Table 8

Summary of the HERMES target performance in the years 1997, 2000 and 2002. The tabulated values are averaged over the respective running periods. The $\Delta P_{i}$ 's represent the polarization losses arising from the listed process (see text).

and deuterium target leads to the following conclusions. At the same guide field, in the case of deuterium the spin exchange and wall relaxation processes are suppressed by a factor $\left(B_{C}^{H} / B_{C}^{D}\right)^{2} \simeq 20$ compared to hydrogen. The positive and negative $P_{z}$ values for hydrogen coincide very well, while for deuterium there is a clear difference. This is due to the larger number of efficiencies and transmissions involved.

From the table it is possible to deduce departures from the optimal performance like the amount of recombination $\left(\Delta \alpha_{r}=1-\alpha_{r}\right)$, and the depolarization due to the various depolarizing mechansims: spin-exchange collisions $\left(\Delta P_{S E}\right)$, wall-depolarization $\left(\Delta P_{W D}\right)$ and beam-induced fields $\left(\Delta P_{B I}\right)$. The target density integrated over the cell length, or areal density $t$, is also shown together with a relative factor of merit (FOM) that takes into account both the polarization and the density. It is worthwhile to mention again that in 1997 the target cell had a larger cross-section $(29 \mathrm{~mm} \times 9.8 \mathrm{~mm})$ implying a higher conductance and a correspondingly lower density (about 40\%). The table also illustrates once more the optimal performance of the $D_{\|}$target in 2000. For this target no recombination $\left(\Delta \alpha_{r}=0\right)$ and no depolarization phenomena $\left(\Delta P_{W D}=\Delta P_{S E}=\Delta P_{B I}=0\right)$ have been observed. This excellent behavior has to be attributed to the lower critical field of deuterium $(11.7 \mathrm{mT})$ compared to hydrogen $(50.7 \mathrm{mT})$, and to an improvement in the knowledge and preparation of the cell surface coating. The improved surface coating is also responsible for the better performance of the $2002 H_{\perp}$ target compared 
Table 9

\begin{tabular}{|c|c|}
\hline target $($ year $)$ & DIS $/$ million \\
\hline \hline$H_{\|}(1996-97)$ & 2.4 \\
\hline$D_{\|}(1998-2000)$ & 9.1 \\
\hline$H_{\perp}(2002-03)$ & 0.7 \\
\hline
\end{tabular}

Deeply inelastic scattering events collected with the three polarized targets after data quality selection. The numbers are given in millions.

to the $1997 H_{\|}$target, as far as the amount of recombination is concerned.

On the other hand, in 2002, due the higher density obtained and the higher number of wall collisions, spin-relaxation effects became more important, causing a loss in polarization of about $11 \%$ and limiting the gain in the factor of merit with respect to the 1997 running due to the increase in density. This indicates how an enhancement in the figure of merit of the target might be obtained by increasing the holding field proportionally with the density, in order to limit the spin relaxation effects.

\section{Selected HERMES results}

In this last Section before the conclusions, some of the measurements which have been carried out at HERMES with the polarized internal gaseous target during the various running periods are mentioned. The number of deeply inelastic scattering events (after data quality selection) collected with the three polarized targets is given in Tab. 9 .

Among the measurements which have been performed using the HERMES detector, we quote the following few: the measurement of the $g_{1}^{p}$ structure function [39]; the measurement of the $g_{1}^{d}$ structure function [40]; the first measurement of the $b_{1}^{d}$ structure function [41]; measurements of the quark-flavour decomposition of the nucleon spin $[42,43]$; the first measurements of single-spin asymmetries in deep-inelastic scattering off both longitudinal and transversely polarized targets $[44,45,46]$ and a first study of the gluon polarization in nucleons [47].

\section{Conclusion}

The HERMES polarized hydrogen/deuterium target has been reliably operating since 1996. It provides polarization values between 0.78 and 0.85 , densities $1-2 \times 10^{14}$ nucleons $/ \mathrm{cm}^{2}$, and total systematic uncertainties of only $0.03-0.04$. 
The systematics of both the recombination and depolarization processes in the storage cell are fully under control.

\section{Acknowledgements}

We gratefully acknowledge the DESY management for its support and the staff at DESY and the collaborating institutions for their significant effort. This work was supported by the FWO-Flanders, Belgium; the Natural Sciences and Engineering Research Council of Canada; the National Natural Science Foundation of China; the INTAS and ESOP network contributions from the European Community; the German Bundesministerium für Bildung und Forschung; the Deutsche Forschungsgemeinschaft (DFG); the Deutscher Akademischer Austauschdienst (DAAD); the Italian Istituto Nazionale di Fisica Nucleare (INFN); Monbusho International Scientific Research Program, JSPS, and Toray Science Foundation of Japan; the Dutch Foundation for Fundamenteel Onderzoek der Materie (FOM); the U. K. Engineering and Physical Sciences Research Council and the Particle Physics and Astronomy Research Council; and the U. S. Department of Energy and the National Science Foundation.

\section{References}

[1] HERMES Technical Design Report, DESY-PRC 93/06, 1993.

[2] K. Rith, Progr. Part. Nucl. Phys 49 (2002) 245.

[3] A. A. Sokolov and I. M. Ternov, Sov. Phys, Doklady 8 (1964) 1203.

[4] D. P. Barber et al., Phys. Lett. B 343 (1995) 436.

[5] K. Ackerstaff et al., Nucl. Instr. Meth. A 417 (1998) 230.

[6] E. Steffens and W. Haeberli, Rep. Prog. Phys. 66 (2003) 1887.

[7] D. De Schepper et al., Nucl. Instrum Meth. A 419 (1998) 16.

[8] A. Nass, et al., Nucl. Inst. and Meth. A 505 (2003) 633.

[9] C. Baumgarten et al., Nucl. Inst. and Meth. A 496 (2003) 277.

[10] C. Baumgarten et al., Nucl. Inst. and Meth A 508 (2003) 268.

[11] C. Baumgarten et al., Nucl. Inst. and Meth. A 482 (2002) 606.

[12] C. Baumgarten et al., Nucl. Inst. and Meth A 496 (2003) 263.

[13] K. Ackerstaff et al., Phys. Rev. Lett. 82 (1999) 1164. 
[14] A. Airapetian et al., Eur. Phys. J. D. 29 (2004) 21.

[15] C. Baumgarten et al., Eur. Phys. J. D 18 (2002) 37.

[16] C. Baumgarten, Ph.D. Thesis, University of Munich, Germany, DESY-THESIS2000-038 (2000).

[17] G. E. Thomas et al., Nucl.Instrum. and Meth. A 257 (1987) 32.

[18] M. A. Bouchiat, J. Phys. Radium 24 (1963) 379.

[19] M. A. Bouchiat, J. Phys. Radium 24 (1963) 611.

[20] M. A. Bouchiat and J. Brossel, Phys. Rev. 147 (1965) 41.

[21] P. W. Anderson in Magnetism vol. I, G. T. Radio and H. Suhl (Edts.), Academic Press N. Y. (1963).

[22] N. Koch and E. Steffens, Rev. Sci. Instrum. 70 (1999) 1631.

[23] K. Halbach, Nucl.Instrum. and Meth. A 169 (1980) 213.

[24] D.Reggiani, Ph.D. thesis, University of Ferrara, Italy, DESY-THESIS-2003-001 (2003).

[25] S. Holloway, Surf. Sci. 299/300 (1994) 656.

[26] T. Engel and G. Ertl, J. Chem. Phys. 69 (1978) 1267.

[27] H. Conrad, G. Ertl and J. Küppers, Surf. Sci. 76 (1978) 323.

[28] E. W. Kuipers et al., Phys. Rev. Lett. 66 (1991) 116.

[29] C. T. Rettner, Phys. Rev. Lett. 69 (1992) 383.

[30] B. Braun, Ph.D thesis MPIH - V25, Ludwig Maximilians-University, Munich, Germany (1995).

[31] J. S. Price and W. Haeberli, Nucl. Instr. Meth. A 326, (1993) 416.

[32] J. S. Price and W. Haeberli, Nucl. Instr. Meth. A 349, (1994) 321.

[33] H. Kolster, Ph.D. thesis, Ludwig Maximilians-University, Munich, Germany (1998).

[34] E. M. Purcell and G.B. Fields, Astrophys. J. 124 (1956) 542.

[35] M. Desainfuscien and C. Auodoin, Phys. Rev. A 13 (1976) 2070.

[36] A. C. Allison, Phys. Rev. A 5 (1972) 2695.

[37] J. F. J. van den Brand et al., Phys. Rev. Lett. 78 (1997) 1235.

[38] T. Wise et al., Phys. Rev. Lett. 87 (2001) 042701.

[39] A. Airapetian et al., Phys. Lett. B 442 (1998) 484. 
[40] C. Weiskopf, Proceedings of the IX International Conference on Deep Inelastic Scattering and QCD (DIS 2001), Bologna - Italy, 2001.

[41] M. Contalbrigo, Proceedings of XV International Spin Physics Symposium (SPIN 2002), Brookhaven National Laboratory - NY (USA), 2002

[42] K. Ackerstaff et al., Phys. Lett. B 464 (1999) 123.

[43] A. Airapetian et al., Phys. Rev. Lett. 92 (2004) 012005.

[44] A. Airapetian et al., Phys. Rev. Lett. 84 (2000) 4047.

[45] A. Airapetian et al., Phys. Rev. B. 562 (2003) 182.

[46] A. Airapetian et al., submitted to Phys. Rev. Lett.; hep-ex/0408013

[47] A. Airapetian et al., Phys. Rev. Lett. 84 (2000) 2584. 